\title{
Intrinsic Absorption in the Spectrum of NGC 7469: Simultaneous Chandra, FUSE and STIS Observations
}

\author{
Jennifer E. Scott ${ }^{1}$, Gerard A. Kriss ${ }^{2,3}$, Julia C. Lee ${ }^{4,5}$, Jessica Kim Quijano ${ }^{2}$, Michael \\ Brotherton $^{6}$, Claude R. Canizares ${ }^{7}$, Richard F. Green ${ }^{8}$, John Hutchings ${ }^{9}$, Mary Elizabeth \\ Kaiser $^{3}$, Herman Marshall ${ }^{7}$, William Oegerle ${ }^{10}$, Patrick Ogle ${ }^{11}$, \& Wei Zheng ${ }^{3}$
}

\begin{abstract}
We present simultaneous X-ray, far-ultraviolet, and near-ultraviolet spectra of the Seyfert 1 galaxy NGC 7469 obtained with the Chandra X-Ray Observatory, the Far Ultraviolet Spectroscopic Explorer, and the Space Telescope Imaging Spectrograph on the Hubble Space Telescope. Previous non-simultaneous observations of this galaxy found two distinct UV absorption components, at -560 and $-1900 \mathrm{~km} \mathrm{~s}^{-1}$, with the former as the likely counterpart of the X-ray absorber.
\end{abstract}

\footnotetext{
${ }^{1}$ Observational Cosmology Laboratory, National Aeronautics and Space Administration, Goddard Space Flight Center, Greenbelt, MD 20771 USA; jscott@stis.gsfc.nasa.gov

${ }^{2}$ Space Telescope Science Institute, 3700 San Martin Drive, Baltimore, MD 21218 USA; [gak,jkim]@stsci.edu

${ }^{3}$ Center for Astrophysical Sciences, Department of Physics and Astronomy, The Johns Hopkins University, Baltimore, MD 21218 USA; [kaiser,zheng] @pha.jhu.edu

${ }^{4}$ Harvard-Smithsonian Center for Astrophysics, 60 Garden Street, MS-4, Cambridge, MA 02138 USA; jclee@cfa.harvard.edu

${ }^{5}$ Chandra Fellow

${ }^{6}$ Department of Physics and Astronomy, University of Wyoming, Laramie, WY, 82071 USA; mbrother@uwyo.edu

${ }^{7}$ Department of Physics and Center for Space Research, Massachusetts Institute of Technology, 77 Massachusetts Avenue, NE80, Cambridge, MA 02139 USA; [crc,hermanm]@space.mit.edu

${ }^{8}$ Kitt Peak National Observatory, National Optical Astronomy Observatories, P.O. Box 26732, 950 North Cherry Avenue, Tucson, AZ 85726 USA; rgreen@noao.edu

${ }^{9}$ Herzberg Institute of Astrophysics, National Research Council of Canada, Victoria, BC V9E 2E7, Canada; john.hutchings@hia.nrc.ca

${ }^{10}$ ExoPlanets and Stellar Astrophysics Laboratory, National Aeronautics and Space Administration, Goddard Space Flight Center, Greenbelt, MD 20771 USA; oegerle@uvo.gsfc.nasa.gov

11 Mail Code 238-332, Jet Propulsion Lab, 4800 Oak Grove Drive, Pasadena, CA 91109 USA; pmo@sgra.jpl.nasa.gov
} 
We confirm these two absorption components in our new UV observations, in which we detect prominent O VI, Ly $\alpha, \mathrm{N}$ V, and C IV absorption. In our Chandra spectrum we detect O VIII emission, but no significant O VIII or O VII absorption. We also detect a prominent $\mathrm{Fe} \mathrm{K} \alpha$ emission line in the Chandra spectrum, as well as absorption due to hydrogen-like and helium-like neon, magnesium, and silicon at velocities consistent with the $-560 \mathrm{~km} \mathrm{~s}^{-1} \mathrm{UV}$ absorber. The FUSE and STIS data reveal that the $\mathrm{H}$ I and $\mathrm{C}$ IV column densities in this UV- and X-ray- absorbing component have increased over time, as the UV continuum flux decreased. We use measured H I, N V, C IV, and O VI column densities to model the photoionization state of both absorbers self-consistently. We confirm the general physical picture of the outflow in which the low velocity component is a highly ionized, high density absorber with a total column density of $10^{20} \mathrm{~cm}^{-2}$, located near the broad emission line region, although due to measurable columns of $\mathrm{N} \mathrm{V}$ and C IV, we assign it a somewhat smaller ionization parameter than found previously, $U \sim 1$. The high velocity $U V$ component is of lower density, $\log N=18.6$, and likely resides farther from the central engine as we find its ionization parameter to be $U=0.08$.

Subject headings: galaxies: active — galaxies: individual (NGC 7469) — galaxies: Seyfert — quasars: absorption lines — ultraviolet: galaxies — X-ray: galaxies

\section{Introduction}

Absorption edges are visible in the X-ray spectra of about one half of all low redshift AGN (Reynolds 1997; George et al. 1998; Crenshaw, Kraemer, \& George 2003). Ensemble studies of the warm absorber population in Seyfert galaxies (Blustin et al. 2005) indicate, from estimates of the radial positions of the absorbers, that these systems are likely predominately outflows from the dusty torus surrounding the accretion disk in each object, while those seen in PG quasars are more likely to arise in an accretion disk wind. All of these objects also show high-ionization absorption lines in their UV spectra (Crenshaw et al. 1999). This suggests a connection between the two phenomena, if not a complete identification of one with the other. The position of the absorbing complexes in velocity space, generally blueshifted with respect to the systemic redshift of the parent AGNs, the variability of the absorption (Kriss et al. 1995; Shields \& Hamann 1997; Crenshaw \& Kraemer 1999; Crenshaw et al. 2000; Kraemer et al. 2001, 2002; Gabel et al. 2003a) and the non-unity continuum and/or broad emission line region (BELR) covering fractions determined for many UV absorbers (Kriss et al. 2000b; Gabel et al. 2003b; Kraemer, Crenshaw, \& Gabel 2001; Kraemer 
et al. 2001; Kraemer et al. 2003; Brotherton et al. 2002; Crenshaw et al. 2003) point to an origin for the gas that is intrinsic to the AGN. AGN outflows are a potentially significant source of feedback energy (Granato et al. 2004; Scannapieco \& Oh 2004), influencing the luminosity-temperature relation for the intracluster medium of galaxy clusters by steepening it on the scale of galaxy groups (Cavaliere et al. 2002), as well as providing metals to and governing the formation of galaxies and later generations of AGNs from the intergalactic medium (Adelberger et al. 2003).

Intrinsic absorption in many AGNs has been studied using high spectral resolution UV and X-ray observations: Mrk 279 (Scott et al. 2004; Gabel et al. 2005); Mrk 509 (Kriss et al. 2000b; Kraemer et al. 2003; Yaqoob et al. 2003); NGC 3516 (Kraemer et al. 2002); NGC 3783 (Kraemer, Crenshaw, \& Gabel 2001; Kaspi et al. 2001; Blustin et al. 2002; Gabel et al. 2003a,b); NGC 4051 (Collinge et al. 2001; Ogle et al. 2004); NGC 4151 (Crenshaw et al. 2000; Kraemer et al. 2001); NGC 5548 (Mathur et al. 1999; Crenshaw \& Kraemer 1999; Brotherton et al. 2002; Kaastra et al. 2000, 2002; Crenshaw et al. 2003; Steenbrugge et al. 2003). However, with the exception of Mrk 279 and some data taken for the longterm campaign on NGC 3783, these observations have been performed at different times in the UV and X-rays. The high degree of variability in AGN continua complicates selfconsistent photoionization modeling of the intrinsic absorption from these non-simultaneous data, preventing firm conclusions about the nature of the relationship between UV and X-ray absorbers.

The Seyfert 1 galaxy NGC 7469 ( $z=0.01639$, de Vaucouleurs et al. 1991) has been studied extensively in the optical, ultraviolet, and X-ray regimes (Wanders et al. 1997; Collier et al. 1998), and has been the subject of simultaneous UV and X-ray variability studies (Kriss et al. 2000a; Nandra et al. 1998, 2000). The intrinsic absorption in NGC 7469 has been studied previously in the X-ray with XMM-Newton (Blustin et al. 2003) and in the UV with FUSE (Kriss et al. 2003). However those observations were separated by one year, making the campaign presented here the first set of simultaneous, high-resolution UV and X-ray spectral observations. These authors found two primary components in the UV, with outflow velocities of -569 and $-1898 \mathrm{~km} \mathrm{~s}^{-1}$. The low velocity component is identified with the high-ionization phase of the X-ray absorption, also responsible for the X-ray emission. Its partial covering fraction suggests its position coincides with that of the BELR. The high velocity UV component has no associated X-ray absorber. Likewise, the low-ionization phase of the X-ray absorption appears to have no UV counterpart.

Here, we present the simultaneous UV and X-ray observations and describe the properties of the AGN outflow. We present photoionization models of the absorbing gas and draw conclusions about its geometry. 


\section{X-ray: Chandra}

\subsection{Data}

We observed NGC 7469 on 2002 December 12-13 for $150 \mathrm{ksec}$ with the High Energy Transmission Grating Spectrometer (HETGS) on the Chandra X-Ray Observatory. The HETGS ${ }^{1}$ provides resolving power up to $\sim 1000$ from $0.5 \mathrm{keV}$ to $8.0 \mathrm{keV}$ through the use of two gratings: (1) the Medium Energy Grating (MEG) which gives good spectral coverage from $0.5 \mathrm{keV}$ to $5.0 \mathrm{keV}(2.5-25 \AA)$; and (2) the High Energy Grating (HEG), which is optimized for the $0.8-8.0 \mathrm{keV}(1.6-15 \AA)$ spectral region.

We extracted the spectra using the standard CIAO v3.0.1 pipeline. In order to maximize signal-to-noise, we co-added the \pm 1 order MEG and HEG spectra and binned to $0.02 \AA$ for analysis. Coarser $0.06 \AA$ bins were used to determine the continuum model. We used ISIS v.1.2.12 (Houck \& Denicola 2000) for spectral analysis. See Table 1 for a summary of the Chandra observation details.

\subsection{Analysis: Continuum Fit and Spectral Features}

We fit the coarsely-binned MEG and HEG spectra in the 0.5-8.5 keV range with a powerlaw plus blackbody continuum modified by absorption from a Galactic hydrogen column of $4.8 \times 10^{20} \mathrm{~cm}^{-2}$ (Dickey \& Lockman 1990). We used the XSPEC models tbabs (Wilms, Allen, $\& \mathrm{M}^{\mathrm{c}}$ Cray 2000) and diskbb (see, eg. Mitsuda et al. 1984; Makishima et al. 1986), respectively to model the absorption and black-body components. The photon index of the power-law spectrum is $\Gamma=1.7910 \pm 0.0009$, with a normalization of $A=(6.97 \pm 0.10) \times 10^{-3}$ photons $\mathrm{cm}^{-2} \mathrm{~s}^{-1} \mathrm{keV}^{-1}$ at $1 \mathrm{keV}$ in the emitted frame, and the temperature of the blackbody is $120 \pm 5 \mathrm{eV}$, with overall $\chi^{2} / d o f=1.12$. As Blustin et al. (2003), we note that this continuum model is simply a convenient parametrization that allows us to fit the emission and absorption features in the Chandra spectrum, it is not meant to be a physical model of the continuum. The errors quoted are 90\% confidence limits. The HETG spectrum, the continuum fit, and its components, including a Gaussian fit to the $\mathrm{Fe} \mathrm{K} \alpha$ line, are shown in Figure 1. We also included additional multiplicative model components to account for calibration effects. We use: (1) an ad hoc correction for ACIS pileup effects which also reduces the effect of the iridium M-edge (Marshall et al. 2004a); (2) a correction to bring the frontside-illuminated

\footnotetext{
${ }^{1}$ For further details on Chandra instruments, see http://cxc.harvard.edu/proposer/POG.

${ }^{2}$ http://space.mit.edu/ASC/ISIS/
} 
chip QEs into agreement with the backside-illuminated chip quantum efficiencies (Marshall et al. 2004a); and (3) an ACIS contamination correction (Marshall et al. 2004b).

In regions of overlap, we require a spectral feature to be identified in both the HEG and MEG spectra in order to qualify as a bona fide absorption or emission line. Because the resolution of the HEG spectrum is $2 \times$ that of the MEG spectrum, we use the HEG spectrum alone to measure the centroid equivalent width(flux) and FWHM of an absorption(emission) line in the 1.6-12.6 $\AA$ region. At longer wavelengths, the $\mathrm{S} / \mathrm{N}$ in the HEG spectrum falls below $\sim 3$ and we use the MEG spectrum. We also require reasonable oscillator strengths for quoted absorption features. For example, if we detect a Ly $\beta$ feature from a particular species, we require its Ly $\alpha$ transition to be detected as well. The HEG and MEG spectra are shown in detail, with the absorption and emission lines we identify and describe below, in Figures 2-4. The continuum fit shown in these figures is the global fit discussed above. Because of the low count rate in the ACIS pixels, we derive the flux errors per pixel from $\sigma_{N} \approx 1+\sqrt{N+\frac{3}{4}}$ where $N$ is the count rate (Gehrels 1986), in order to approximate Gaussian statistics. For comparison we also show the $X M M /$ RGS spectrum from Blustin et al. (2003), in the regions of overlap with the Chandra/HETG data, $6-25 \AA$, in Figures 3 and 4 .

We find several absorption features in the Chandra/HETG spectra, and we fit Gaussians to the unresolved profiles to derive the equivalent widths, the line centroids, and the velocity widths. We find Ly $\alpha$ lines of the hydrogen-like ions $\mathrm{Ne} \mathrm{X}$ and $\mathrm{Mg} \mathrm{XII}$, and a marginal Si XIV feature (Figure 5) and the He $\alpha$ lines of Ne IX, Mg XI, and Si XIII (Figure 6). We also detect $\mathrm{Ne}$ IX $\mathrm{He} \beta$ with an outflow velocity and line width consistent with the He $\alpha$ line. Of these features, Blustin et al. (2003) find only Ne IX He $\beta$ in their $X M M /$ RGS spectrum of NGC 7469. However we note that the resolution of the RGS is lower than that of both the HEG and the MEG, by factors of $\sim 2.5$ and $\sim 5$ respectively. Also, the Si XIV line lies at the high energy edge of the RGS spectral range. We also find three iron features, Fe XVII $\lambda 15.0$, Fe XXI $\lambda 12.3$, and Fe XX $\lambda 12.8$. No Fe XVII absorption is not found in the XMM/RGS data, but there is an ambiguous feature at the position of Fe XXI. The Fe XX feature falls on a gap in the data. Conversely, Blustin et al. (2003) report several absorption features that we do not find, or find only marginal evidence for, in the Chandra spectra: O VIII Ly $\alpha$ and Ly $\beta$ which we discuss further below, O VII 3-1 (r) (18.6 $\AA)$, and Ne VII Be $\alpha$ (13.8 $\AA$ ). The other lines reported by these authors lie in regions where the HETGS effective area is low, or outside its spectral range altogether.

The parameters of the absorption line fits are summarized in Table 2, as well as those for the emission features discussed below. Along with the equivalent widths, line centroids, and line widths, we tabulate the statistical significance of each feature defined by the detection 
threshold. This is defined as the equivalent width divided by the the $1 \sigma$ equivalent width error, calculated as a function of wavelength using the spectrum flux and error boxcarsmoothed over $\sim 2.5 \times$ the FWHM of the line spread function (Bechtold et al. 2002). We list all the absorption and emission lines we detect at greater than $\sim 2.5 \sigma$ in Table 2, including those for which we could make no identification. The low confidence level we set leaves open the possibility that some of the features in Table 2 are spurious.

We find a marginal narrow Ly $\alpha$ emission feature redward of the Ne X absorption, shown in the top panel of Figure 5. The significance of both the Ne X absorption and emission lines in Table 2 is low, $1.3 \sigma$, and they are not detected in the $X M M /$ RGS spectrum of Blustin et al. (2003). However, we do detect them at $4.1 \sigma$ and $3.6 \sigma$ in the MEG spectrum.

We see some evidence of O VIII absorption blueward of, and possibly filled in by the Ly $\alpha$ emission line, as reported by Blustin et al. (2003), but we do not find O VIII Ly $\beta$ or Ly $\gamma$ absorption in the Chandra spectrum (Figure 7) as Blustin et al. (2003) did. We do not find $\mathrm{O}$ VIII $\mathrm{Ly} \beta, \operatorname{Ly} \gamma$, or $\operatorname{Ly} \delta$ emission corresponding to the prominent Ly $\alpha$ emission line, but we do find significant emission features near the expected positions of the Ly-5, Ly-6, and Ly-7 lines. However, because the implied velocities of these features are not entirely consistent with the Ly $\alpha$ line, these are marked as highly tentative identifications in Table 2 and in Figure 7. These lines are not seen in the $X M M /$ RGS spectrum.

From the $\mathrm{S} / \mathrm{N}$ in the spectrum at the expected position of $\mathrm{O}$ VIII Ly $\alpha$ absorption, we place a $3 \sigma$ upper limit of $\sim 30 \mathrm{~m} \AA$ on its equivalent width, which implies $N(\mathrm{O}$ VIII $)<$ $8.8 \times 10^{17} \mathrm{~cm}^{-2}$ for $b \sim 100 \mathrm{~km} \mathrm{~s}^{-1}$. We find no O VII He $\alpha$ absorption, leading to an upper limit on the equivalent width of $80 \mathrm{~m} \AA$, or $N(\mathrm{O}$ VII $)<1.4 \times 10^{19} \mathrm{~cm}^{-2}$, also for $b \sim 100 \mathrm{~km} \mathrm{~s}^{-1}$. This choice of Doppler parameter derives from the identification of this unresolved X-ray absorption with Component 1 in the UV spectra, which we model with nine subcomponents with $\mathrm{FWHM}=61 \mathrm{~km} \mathrm{~s}^{-1}$, as described in Section 3.2.3. If we instead assume $b=200 \mathrm{~km} \mathrm{~s}^{-1}$, the O VIII and O VII curves of growth give $N(\mathrm{O}$ VIII $)<5.1 \times 10^{16} \mathrm{~cm}^{-2}$ and $N(\mathrm{O}$ VII $)<3.4 \times 10^{18} \mathrm{~cm}^{-2}$, while $b=500 \mathrm{~km} \mathrm{~s}^{-1}$ gives $N(\mathrm{O}$ VIII $)<2.8 \times 10^{16} \mathrm{~cm}^{-2}$ and $N(\mathrm{O}$ VII $)<5.8 \times 10^{16} \mathrm{~cm}^{-2}$. These dramatically lower O VII column densities do not change the conclusions we draw from the photoionization models in Section 4.

An emission feature consistent with O VII forbidden at $-200 \mathrm{~km} \mathrm{~s}^{-1}$ is shown in the top panel of Figure 8. At $\sim 110 \mathrm{~m} \AA$, it is stronger than reported by Blustin et al. (2003). The corresponding intercombination line, however, is highly uncertain if it is present at all, as shown in the bottom panel of the figure. Blustin et al. (2003) report the forbidden to intercombination ratio $\mathrm{f} / \mathrm{i} \sim 2$ for $\mathrm{O}$ VII in the $X M M$ data from 2000 . If that ratio held for this observation, the $\mathrm{S} / \mathrm{N}$ in the Chandra spectrum is such that we would only expect to find the intercombination line at $\sim 2.5 \sigma$ confidence. We find a significant emission feature near 
the expected position of the O VII radiative recombination continuum, listed in Table 2. The emission feature in the top panel of Figure 8 consistent with $\mathrm{O}$ VII(f) at $-980 \mathrm{~km} \mathrm{~s}^{-1}$ is not statistically significant. Finally, we identify a significant emission feature at $13.91 \AA$ observed with $\mathrm{Ne}$ IX(f) at $-200 \mathrm{~km} \mathrm{~s}^{-1}$, although, again, we find no corresponding resonance or intercombination lines.

A strong $\mathrm{Fe} \mathrm{K} \alpha$ emission line is present in the Chandra spectrum. BeppoSAX observations (De Rosa et al. 2002) indicated the presence of a narrow emission component in addition to the broad emission from an accretion disk. However, Blustin et al. (2003) found that the $\mathrm{Fe} \mathrm{K} \alpha$ emission line in their $X M M$ spectrum was best fitted by a single narrow Gaussian. Using the continuum defined above and fitting only the 1.5-2.5 $\AA$ region of the HEG spectrum, we find a single Gaussian with flux $3.9 \pm 0.7 \times 10^{-5}$ photons $\mathrm{cm}^{-2} \mathrm{~s}^{-1}$, line width $6310 \pm 1580 \mathrm{~km} \mathrm{~s}^{-1}$ (FWHM), and rest-frame energy $6.39 \pm 0.01 \mathrm{keV}$ to be the best fit to this line with $\chi^{2} / d o f=62.5 / 57$. Adding a second, narrow Gaussian does not improve the fit. These parameters are consistent with the $X M M$ results. The width is better constrained by these Chandra data, however, and its value is consistent with the UV emission lines we fit in the STIS spectra, particularly the intermediate velocity width lines of Ly $\alpha, \mathrm{C}$ IV, and N V. These are discussed in the next section. We note that Nandra et al. (2000) found a significant correlation of the flux in the $\mathrm{Fe} \mathrm{K} \alpha$ line and the $2-10 \mathrm{keV}$ continuum flux, indicating that at least some of the flux arises within 1 light-day of the continuum source.

\section{Ultraviolet: FUSE and STIS}

\subsection{Data}

We observed NGC 7469 with FUSE using the $30^{\prime \prime} \times 30^{\prime \prime}$ low-resolution aperture. See Table 1 for the details of the observations. We combined the two exposures taken on 2002 December 13 for a total exposure time of $6948 \mathrm{sec}$. Because of visibility constraints imposed by the coordinated observation, there was insufficient time to align the SiC1, SiC2, and LiF2 channels for the FUSE exposures. Our method for correcting for the worm feature in the LiF1B segment uses the shape and flux level of the target in the LiF2A channel, therefore we were not able to perform the correction to derive a reliable spectrum over 1093-1182 $\AA$.

We used the standard FUSE calibration pipelines (CALFUSE v2.2.3, see Sahnow et al. 2000 ), to extract the spectra and to perform background subtraction and wavelength and flux calibrations. We combined the spectra from each detector and binned the spectra to $0.05 \AA$ bins, improving the $\mathrm{S} / \mathrm{N}$ while preserving the full spectral resolution, $\sim 20 \mathrm{~km} \mathrm{~s}^{-1}$.

To correct the wavelength scale of the FUSE spectra for zero-point offsets due to place- 
ment of the target in the $30^{\prime \prime}$ aperture, we applied a $0.0638 \AA$ shift to bring the wavelength scale into agreement with the heliocentric spectrum observed by FUSE in 1999 described by Kriss et al. (2003). These authors established the zero point of the wavelength scale by comparing the positions of interstellar absorption lines such as Ar I, Fe II, O I, and $\mathrm{H}_{2}$ to the velocity of the Galactic $21 \mathrm{~cm}$ emission. We estimate that the residual systematic errors in the FUSE wavelength scale are on the order of $15 \mathrm{~km} \mathrm{~s}^{-1}$, and that the flux scale is accurate to $\sim 10 \%$.

Simultaneously with the FUSE observations, we obtained observations of NGC 7469 with the STIS FUV MAMA over 5 orbits $(13013 \mathrm{sec})$ through the $0.2^{\prime \prime} \times 0.2^{\prime \prime}$ aperture using the medium-resolution E140M echelle grating covering 1150-1730 $\AA$. We also obtained 9 orbits (22810 sec) of STIS data on NGC 7469 over two visits on 2004 June 21-22 using the same set-up as above. See Table 1 for other details of the STIS observations. We used CALSTIS v2.13b to process the spectra. As described above, we measure the positions of lines from low-ionization species, Si II, N I, Si III, C II, C II*, Si IV, Fe II, and Al II in the STIS spectrum, and compare these to the mean heliocentric velocity of the H I 21$\mathrm{cm}$ emission. We find that no correction to the wavelength scale derived by the CALSTIS pipeline is necessary. Using a white dwarf spectrophotometric standard, we applied a small correction to the flux in the STIS spectra to correct for residual echelle ripple structure and changes in the MAMA detector sensitivity not accounted for in the current STIS pipeline. Gabel et al. (2005) will present a full description of this process.

Finally, we discuss the origin of a depression in the STIS spectrum at $1418.8 \AA$. The expected position of redshifted Si IV at rest with respect to the AGN is $1416.61 \AA$, so this feature is inconsistent with Si IV absorption due to intrinsic, outflowing gas. Also, this feature is shallow and broad, and qualitatively unlike other intrinsic features. We attribute this feature to an instrumental effect, namely a shadow on the MAMA detector. The STIS FUV MAMA employs a field electrode, or repeller wire, to direct any electrons emitted in directions away from the microchannel plate detectors back into the channels. The repeller wire shadow runs from $(x, y)=(1,994)$ to $(1048,1134)$. The feature appears in order 104 , which is centered at $y=1030.7$ in pixel space, and runs from $y=1038$ to $y=1014$, covering wavelengths 1414.3 to $1429.9 \AA$. The wire crosses the dispersion axis at an obtuse angle, thus the feature is broad in the extracted spectrum. Because the shadow appears in slightly different locations depending which optical element is present in the spectrograph, it is not divided out in the flat field, but simply flagged in the data quality array. A broad depression in the flux in STIS/E140M spectra has been reported by others (Kraemer et al. 2001; Leitherer et al. 2001), and a very similar feature is visible in an E140M spectrum of Mrk 279 (Gabel et al. 2005). Therefore, we conclude that this is not a feature intrinsic to NGC 7469. 


\subsection{Analysis}

\subsubsection{Continuum and Emission Lines}

We used the IRAF ${ }^{3}$ task specfit (Kriss 1994) and spectral regions unaffected by absorption features to fit the continuum and emission lines in the FUSE and STIS spectra of NGC 7469. This routine is an interactive spectral fitting tool that uses $\chi^{2}$ minimization techniques to derive the best fitting parameters for a user-specified model. For the continuum, we fit a power law of the form $f_{\lambda}=f_{1000} \lambda^{-\alpha}$ to the 2002 FUSE and STIS spectra simultaneously, where the normalization, $f_{1000}$, is the flux at $1000 \AA$, and we include the Galactic extinction law of Cardelli, Clayton \& Mathis (1989) with $\mathrm{R}_{V}=3.1$ and $\mathrm{E}(\mathrm{B}-\mathrm{V})=0.069$ (Schlegel, Finkbeiner, \& Davis 1998) in the continuum fits. The best fit parameters are $\alpha=1.082 \pm 0.021$ and $f_{1000}=8.18 \pm 0.06 \times 10^{-14} \mathrm{ergs} \mathrm{s}^{-1} \mathrm{~cm}^{-2} \AA^{-1}$. For the 2004 STIS spectrum, we fit $f_{\lambda}=3.25 \pm 0.01 \times 10^{-14} \lambda^{-1.086 \pm 0.007} \mathrm{ergs} \mathrm{s}^{-1} \mathrm{~cm}^{-2} \AA^{-1}$. We fit Gaussian profiles to the emission lines in all the spectra, the most prominent of which are broad and narrow components of O VI in the FUSE band, and Ly $\alpha$ and C IV in the STIS band. The Ly $\alpha$ profile requires four Gaussian components for a reasonable fit- broad, intermediate and two narrow components. The C IV emission line requires one broad, one intermediate, and one narrow component for a good fit, and we fit intermediate width and narrow components to the $\mathrm{N}$ V emission. These emission lines are plotted in Figures 9 and 10, and the fit parameters are tabulated in Table 3.

\subsubsection{Interstellar and Intergalactic Absorption Features}

There are 4 velocity components of 21-cm emission along the NGC 7469 sightline, including one high velocity component associated with the Magellanic Stream at $-333 \mathrm{~km} \mathrm{~s}^{-1}$ (Wakker et al. 2001). The strongest component of the interstellar absorption, with strong molecular hydrogen absorption (Wakker et al. 2003), lies at zero velocity. We divide the ISM absorption from the FUSE spectrum using a two-temperature model of the zero velocity absorption constructed from the FUSE spectral simulation code FSIM. Because the STIS spectra are much less crowded, we can use an empirical approach, modeling the ISM lines in the vicinity of intrinsic lines by fitting unblended lines of the same species and scaling by the appropriate oscillator strength ratios from published atomic data.

\footnotetext{
${ }^{3}$ IRAF is distributed by the National Optical Astronomy Observatories, which are operated by the Association of Universities for Research in Astronomy, Inc., under cooperative agreement with the National Science Foundation.
} 
We identify an intergalactic absorption feature at $z=0.0134$ from its strong Ly $\alpha$ line in the STIS spectra. The qualitative shape of this feature is not as broad and shallow as the feature at $1419 \AA$ caused by the repeller wire shadow discussed above, and no such feature is expected at this position, or flagged near this position in the data quality array.

Curiously, this intergalactic Ly $\alpha$ lies between the two components of intrinsic absorption we discuss below. We consider this system intergalactic for the following reasons: (1) We find no O VI, N V, or C IV absorption corresponding to this system; and (2) The absorption does not vary between the 2002 and 2004 observations as the UV flux decreases by a factor of 2.5. This could because its absorption is highly saturated, but we rule out this possibility, even for a small covering fraction because no corresponding Ly $\beta$ absorption is seen in the FUSE spectrum. Fixing the covering fraction to unity, we use specfit to derive $N(\mathrm{H} \mathrm{I})=$ $6.7 \pm 0.3 \times 10^{12} \mathrm{~cm}^{-2}$ and $b=52 \pm 2 \mathrm{~km} \mathrm{~s}^{-1}$ for this intervening absorber.

\subsubsection{Intrinsic Absorption Features}

Kriss et al. (2003) reported two primary components of UV absorption, labeled Components 1 and 2, each with several velocity subcomponents. We use the IRAF task specfit to fit the absorption profiles as Gaussians in optical depth, specified by four parameters: (1) the optical depth at line center, (2) the line centroid, (3) the line width, and (4) the covering fraction. For doublets and members of a line series, we fix the central optical depths to the ratios required by the products of the wavelengths and the oscillator strengths. From these fits, we derive column densities and covering fractions for all subcomponents of the absorption complexes, Components 1 and 2. The errors on these parameters are determined by specfit by evaluating and inverting the curvature matrix around the final value of $\chi^{2}$. For the fits to the absorption profiles, we fix the continuum and emission line fits to those derived from unabsorbed regions, as described above.

Analysis of high S/N spectra by Arav et al. (2002), Gabel et al. (2003, 2005), and Scott et al. (2004) shows that the profiles of intrinsic absorption lines in AGN are best described using a velocity-dependent covering fraction. The FUSE and STIS data for NGC 7469, however, are too noisy for a reliable application of this technique. In addition, because we lack the full Lyman series from the FUSE data and because the C IV and N V doublets are weak in Component 1, as we discuss further below, we do not attempt such a detailed analysis of these data. The next-best approach, as used by Kriss et al. (2003), decomposes the line profile with multiple blended Gaussians. Properly fixed ratios of optical depth in line doublets enables us to constrain the covering fraction in the individual components. Kriss

et al. (2003) found that the quality of the FUSE data were such that a single value of the 
covering fraction adequately described each subcomponent. For consistency, and because our data have even lower $\mathrm{S} / \mathrm{N}$, we adopt the same approach here.

In Component 2, we fit four subcomponents as did Kriss et al. (2003). However, in Component 1, we find blue and red wings of absorption in Ly $\alpha$, leading to a fit consisting of nine subcomponents. We found that our fits did not require any change in the covering fraction of the absorption in either component in either the 2002 or the 2004 epoch observations. We therefore fix the covering fractions to the 1999 values, 0.53 for Component 1 and 0.93 for Component 2. We fix the width of all subcomponents at $61 \pm 4 \mathrm{~km} \mathrm{~s}^{-1}$ and $48 \pm 5 \mathrm{~km} \mathrm{~s}^{-1}$ (FWHM) for Components 1 and 2, respectively. The resulting outflow velocities and column densities in all the absorption subcomponents are listed in Table 4. For the purposes of our analysis, we use the summed column densities of all the subcomponents in Components 1 and 2. The $N(\mathrm{H} \mathrm{I})$-weighted outflow velocity of Component 1 is $-562 \pm 11 \mathrm{~km} \mathrm{~s}^{-1}$ and that of Component 2 is $-1901 \pm 3 \mathrm{~km} \mathrm{~s}^{-1}$. These are plotted in Figures 11, 12, and 13 for all the species identified in intrinsic absorption in the FUSE and STIS spectra.

The H I absorption is identified solely by the presence of the strong Ly $\alpha$ lines in the STIS spectrum, seen in the top panels of Figures 12 and 13. In the 2002 FUSE spectrum no significant Ly $\beta$ absorption is detected. For Component 1, this is consistent with the low $\mathrm{H} \mathrm{I}$ covering fraction of the system. The $\mathrm{Ly} \beta$ in Component 2 lies at the position of a strong interstellar C II line. See Figure 11.

The O VI doublet is strong in both components. As noted by Kriss et al. (2003), the strengths of the doublet lines in Component 1 are equal, or nearly so as best as can be determined by the signal-to-noise ratio in the 2002 spectrum, which results from an exposure $~ 5.5$ times shorter than the 1999 spectrum. Figure 14 shows both lines of the O VI doublet for Components 1 and 2. It is clear from this figure that in Component 1, the flux ratio in the two line profiles is consistent with one within the uncertainties. The same is true, however, for Component 2 in this spectrum. We performed the initial fit the O VI absorption in Component 1 by fixing the velocity offsets and column density ratios of all the subcomponents with respect to the strongest subcomponent, 1d, to those measured for the strong Ly $\alpha$ absorption in the STIS spectrum. We found that allowing the velocity offsets of the subcomponents to vary from the fiducial Ly $\alpha$ values did reduce the $\chi^{2}$ of the fit enough to justify the extra degrees of freedom, and the values in Table 4 reflect the improved fit. Though we are able to measure the O VI column density in Component 1 formally, by summing all the subcomponents, we treat the measurement as a lower limit, since the higher S/N 1999 FUSE spectrum showed that this component is highly saturated. This limit is shown in Figure 17, which we discuss further below in Section 3.2.4. We note the possibility that the O VI column density is different in these 2002 observations than in the 2000 epoch, 
because the UV continuum level has decreased and because $N(\mathrm{H}$ I) increased from its 1999 value. We see no evidence for damping wings in the $\mathrm{O}$ VI profile, so an increase in $N(\mathrm{O}$ VI) from 1999 would imply that the line is only further saturated and our ability to measure the column density precisely has not improved. We do not assume saturation for Component 2, because in several velocity bins, the flux ratio between the blue and red profiles is significantly greater than one, and again, because the 1999 spectrum indicates that the absorption is only nearly, not fully saturated.

We identify prominent N V and C IV doublets associated with Component 2 in the STIS spectra. These species, including Ly $\alpha$ absorption, are all identified in the 1996 epoch data from the HST/Faint Object Spectrograph and from the International Ultraviolet Explorer presented by Kriss et al. (2000). However, we also identify weak but significant N V and C IV doublets associated with Component 1. These are shown in Figures 12 and 13. While the red lines of each doublet are not obviously present in the spectrum, the blue members of the doubles are visible in at least some subcomponents. We established the significance of these doublets by comparing the $\chi^{2}$ of a fit with no $\mathrm{N}$ V and $\mathrm{C}$ IV to that of a fit which did include these doublets. We fit these systems by fixing the covering fractions and line widths as discussed above. We allowed the column density and line center of subcomponent 1d to vary and fixed the velocity offsets and relative column densities of all the other other subcomponents to those of the O VI subcomponents. For N V, the improvement in the $\chi^{2}$ is dramatic in both the 2002 and 2004 STIS spectra: $\Delta \chi^{2}=152$ and $\Delta \chi^{2}=343$, respectively, for the extra 2 degrees of freedom. For C IV we find $\Delta \chi^{2}=22$ and $\Delta \chi^{2}=201$. From this we conclude that these absorption features are indeed present in the data. The results of all these absorption profile fits are listed in Table 4 and the summed column densities are plotted in Figure 17.

\subsubsection{Variability}

Using previous FOS and STIS data along with the data presented here, we plot the variation of the $1000 \AA$ flux and the UV spectral index in Figure 15. From the 1996 observation to 2002, the $1000 \AA$ flux increased by $26 \%$ while the power law index became harder by a similar factor. Between 2000 and 2002, the $1000 \AA$ flux decreased by a factor of $\sim 1.6$, and by another factor of 2.5 between 2002 and 2004. During this time, the spectral index got marginally softer, as expected, but the change is not significant given the uncertainties.

The intrinsic Ly $\alpha$ and C IV $\lambda 1548$ absorption in the 2004 STIS spectrum is shown in comparison with that in the 2002 spectrum in Figure 16. We plot the total H I, O VI, $\mathrm{C} \mathrm{IV}$, and N V column densities in Components 1 and 2 in Figure 17. The top panel of the 
figure includes the upper limit on $N(\mathrm{H} \mathrm{I})$ from the nondetection of $\mathrm{Ly} \beta$ in the 1999 FUSE spectrum presented in Kriss et al. (2003), as well as the measurements from Ly $\alpha$ in the 2002 and 2004 STIS spectra. Also, in the second panel, we show the $N(\mathrm{O}$ VI) measurements for Component 2 and the lower limits from the saturated absorption in Component 1 in the 1999 and 2002 FUSE spectra.

From Figures 16 and 17, we see that the absorption in Component 2 does not vary in most species, with the exception of an increase in N(H I) between 1999 and the 2002/2004 epochs. In Component 1, the $\mathrm{H} \mathrm{I}$ absorption grows stronger, consistent with a change in the column density of a factor of $\sim 4$ from 1999 to 2002 and again by another factor of 2.3 from 2002 to 2004, as the UV flux decreased by a total of a factor of 4 . The lower limit on $N(\mathrm{O}$ VI) is consistent with no change since the 1999 observation. The C IV column density increased by a factor of 4 between the 2002 and 2004 observations, and the N V column density also increased by a smaller factor as the UV flux decreased by a factor of 2.5. Thus, both velocity components of the intrinsic absorption respond over time to changes in the ionizing flux. In Section 5, we discuss how we use this variability to place limits on the radial distances of the absorbers from the AGN.

\section{Photoionization Models}

We computed photoionization models for the absorbing gas similar to those of Krolik \& Kriss $(1995,2001)$ using the XSTAR photoionization code (Kallman et al. 2000). In Figure 18, we show the source spectral energy distribution used in the models. For SED1, we used the UV and X-ray spectral slopes and normalizations fit to the 2002 epoch spectra of NGC 7469, described above in Sections 2.2 and 3.2.1. For comparison, we show SED2, the SED based on the 2000 epoch FUSE and STIS observations of NGC 7469, presented used by Kriss et al. (2003). As described by Kriss et al. (2003), we assume $f_{\nu} \propto \nu^{-1}$ at long wavelengths for both SED1 and SED2. This spectrum breaks at $2500 \AA$ to $f_{\nu} \propto \nu^{-0.92}$ for SED1 and $f_{\nu} \propto \nu^{-0.75}$ for SED2 to match the 2000 and 2002 epoch FUSE spectra, respectively. The X-ray data are matched by $f_{\nu} \propto \nu^{-0.8}$ for SED1 $f_{\nu} \propto \nu^{-0.7}$ for SED2 between $0.5 \mathrm{keV}$ and $100 \mathrm{keV}$.

We ran models in two two-dimensional grids of total absorber column density and ionization parameter, appropriate for Components 1 and 2: $(\log N, \log U)=(19.5-20.75,-3.0-$ 4.0) and (18.3-19.0, -5.3--0.3), respectively, assuming solar abundances (Grevesse, Noels, \& Sauval 1996) in both cases. We then compared the column densities of the observed species: $\mathrm{H} \mathrm{I}, \mathrm{O}$ VI, N V, C IV in the UV data, and the H-like and He-like Ne, Mg, and Si observed in the Chandra data, as well as limits on O VII and O VIII to the values predicted by the 
models in order to determine the properties of the two velocity components of the intrinsic absorption.

For Component 1, we find that $U=1.0$ is consistent with the measurements of $\mathrm{N} V$ and C IV, with the lower limit on the saturated O VI absorption, and with the upper limits on the O VII and O VIII absorption from their nondetection in the Chandra data, for our assumption of $b=100 \mathrm{~km} \mathrm{~s}^{-1}$, but also for Doppler parameters as large as $\sim 1000 \mathrm{~km} \mathrm{~s}^{-1}$. For the total column density, we find $\log N=20.0$ is a reasonable match to the absorbing columns. For Component 2, we find $(\log N, U)=(18.6,0.08)$ gives the best match with the absorption data.

All of the absorption from H-like and He-like ions in the Chandra data is seen at velocities consistent with the outflow velocity of Component 1. However, the column densities we measure imply larger ionization parameters from the models, $8 \lesssim U \lesssim 12$, implying $\log N>$ 20 , indicating the presence of multiple ionization phases, as found in the warm absorber associated with NGC 3783 (Netzer et al. 2003; Krongold et al. 2003) from spectral fitting to photoionization models. The relative dearth of spectral features in our Chandra spectrum of NGC 7469 precludes such detailed modeling in this case.

\section{Discussion}

These new simultaneous UV and X-ray observations of NGC 7469 allow us to compare the column densities of various species observed in the absorbers with photoionization models in a fully self-consistent manner in order to infer the physical conditions in the outflow. From our multi-epoch UV observations, we use the variability of the ionizing continuum and the absorption to draw conclusions about the distances of the absorbing components from the central engine of the AGN.

Kriss et al. (2003) reported $U=6.0$ for Component 1 . Although this value is consistent with our measurement of O VI in the 2002 FUSE data, and the limits on O VII and O VIII from the Chandra spectrum, it is incompatible with the detection of any N V and C IV absorption in the 2002 STIS spectrum. With the added leverage afforded by the column density measurements of these species, we now estimate $U=1.0$ for this velocity component. This decrease in the ionization parameter is consistent with the decrease in the ionizing flux between the 1999 and 2002 observations, although $U=1$ is somewhat smaller than expected for a constant total column density and a scaling of the number of ionizing photons, which decreases by a factor of $\sim 2$ for the SEDs assumed for the 1999 and 2002 epochs shown in Figure 18. The changes in the H I, C IV, and N V column densities between the 
STIS observations in 2002 and 2004 are also consistent with photoionization changes in the absorber. We have no knowledge of the X-ray continuum at the 2004 epoch, but if we make the simple assumption that the X-ray flux decreases in direct proportion to the UV flux, the column densities of these species are in rough agreement with $\log N=20$ and $U=0.4$. We lack the constraining power of a measurement of $N(\mathrm{O} \mathrm{VI})$ at this epoch.

As Steenbrugge et al. (2003) found for the warm absorber in NGC 5548 and Ogle et al. (2004) found for the warm absorber in NGC 4051, we find a trend of increasing column density with increasing ionization parameter for the species we detect in Component 1 of the NGC 7469 absorber. In Figure 19 we show the ionization parameter at which the abundance of each species peaks versus the equivalent hydrogen column density for that species in Component 1 with a least-squares estimation of the power law fit.

The total hydrogen column densities derived from the Fe XX and Fe XXI lines would imply that the absorber is Compton thick. These columns are derived, as all the points in Figure 19 are, from the measured line equivalent widths using the curve of growth and a Doppler parameter of $100 \mathrm{~km} \mathrm{~s}^{-1}$. We use the fractional abundance of the ion from the photoionization models and the assumed solar abundance of each atomic species. There are two ways the points could move down on Figure 19: (1) a larger Doppler parameter, eg. $b=500 \mathrm{~km} \mathrm{~s}^{-1}$ would yield $N_{\mathrm{H}}(\mathrm{Fe} \mathrm{XX})=2 \times 10^{23} \mathrm{~cm}^{-2}$ and $N_{\mathrm{H}}(\mathrm{Fe} \mathrm{XXI})=3 \times 10^{24} \mathrm{~cm}^{-2}$, however, we note that this would affect all the points in the figure; or (2) an enhanced iron abundance in the absorber, as seen in some AGN broad line regions (Hamann \& Ferland 1999) and accretion disks (Lee et al. 1999, Ballantyne \& Fabian 2001, Bianchi \& Matt 2002, Schurch et al. 2003). In any case, removing the Fe XX and Fe XXI points from the fit does not change the slope or the standard deviation of the regression significantly.

To treat the lower limit on $N_{\mathrm{H}}\left(\mathrm{O}\right.$ VI) and the upper limits on $N_{\mathrm{H}}(\mathrm{O}$ VII $)$ and $N_{\mathrm{H}}(\mathrm{O}$ VIII $)$ we used the IRAF emmethod algorithm appropriate for censored data to perform the linear regression. In order to determine if the regression solution is driven primarily by the low- $\mathrm{U}$ points in Figure 19, we performed the linear fit without the C IV, N V, and O VI points included. The standard deviation of the fit and of the individual fit parameters increases, but within this increased uncertainty, the changes in the fit parameters are not significant. This fit is shown by the dotted line in Figure 19. This relationship between $U$ and $N_{\mathrm{H}}$ illustrated in Figure 19 indicates that, while $U=1.0$ for Component 1 is broadly consistent with the UV data, the absorber is composed of gas with a range of ionization parameters and the absorbing column scales with ionization.

The column density and ionization parameter we infer for Component 2 are similar to the values inferred by Kriss et al. (2003) for the 1996 FOS and IUE observations (Kriss et al. 2000a). This is consistent with a change in the ionization parameter due to a change in 
the ionizing flux, as the number of ionizing photons from SED1 is comparable to the number inferred from the Kriss et al. (2000a) SED. (See Figures 15 and 18.)

We find X-ray absorption in helium-like and hydrogen-like neon, magnesium, and silicon all at velocities consistent UV Component 1 to within $2 \sigma$, although not with the same total column density and ionization parameter as the UV-absorbing species. This supports the conclusions of Kriss et al. (2003) and Blustin et al. (2003), that this component is associated with the X-ray warm absorber and is located closer to the central engine than Component 2. This is further corroborated by the small covering fraction and outflow velocity of Component $1, \sim 0.5$ and $-560 \mathrm{~km} \mathrm{~s}^{-1}$, respectively, compared with the unity covering fraction and $\sim-1900 \mathrm{~km} \mathrm{~s}^{-1}$ velocity of Component 2 with respect to the systemic redshift of NGC 7469. The small covering fraction of Component 1 may indicate full covering of the continuum source and partial covering of the BELR. This geometry is consistent with the larger size of the BELR with respect to the central, continuum emission source, and has also been inferred in the outflow in Mrk 279 by applying simultaneous fits to several Lyman series lines and to the C IV, N V, and O VI doublets (Scott et al. 2004; Gabel et al. 2005). The increase in the H I, N V, and C IV column densities in Component 1 with the decrease in the flux in the ionizing continuum also supports this interpretation.

The only X-ray absorption feature we find in common with the results of Blustin et al. (2003) is the Ne IX $\mathrm{He} \beta$ line at a rest wavelength of $11.5 \AA$ identified in Component 1. We report its equivalent width as $14 \pm 5 \mathrm{~m} \AA$, while Blustin et al. (2003) found $50 \pm 20$ $\mathrm{m} \AA$. The marginal decrease in the Ne IX absorption is consistent with a photoionization effect and the adopted SEDs shown in Figure 18 in the sense that the flux at the ionization potential of Ne VIII is $23 \%$ larger in the 2000 epoch SED (SED1) relative to the 2002 SED (SED2). The total column density in Component 1 implied by UV absorption features from the photoionization modeling discussed in Section $4, \log N=20$, is consistent with that found for the X-ray absorber by Blustin et al. (2003) from their XMM data. However, as noted above, and as illustrated by Figure 19, the hydrogen-like and helium-like absorption we find in the Chandra data imply a higher total column density indicating a range of ionization phases.

We use the fact that we see variability in the absorption in Component 1, particularly in its H I absorption, and the time between the 2002 and 2004 observations to place a 540-day upper limit on the recombination time of the gas, where $t_{r e c} \equiv\left(n_{e} \alpha_{r e c, i} \frac{n_{i+1}}{n_{i}}\right)^{-1}$ (Krolik \& Kriss 1995). For $T=3 \times 10^{4} \mathrm{~K}$, the hydrogen recombination rate is $\sim 2.3 \times 10^{-13} \mathrm{~cm}^{3} \mathrm{~s}^{-1}$. This gives a fairly weak lower limit on the density, $n_{e} \gtrsim 45 \mathrm{~cm}^{-3}$, assuming a neutral fraction of $5 \times 10^{-4}$. Using the ionization parameter $U=1.0$, this gives an upper limit on the radial distance of Component 1 of $\sim 100$ pc. Following the prescription of Blustin et al. 
(2005) we estimate the maximum radial distance under the assumption that the absorption arises in a region of width less than or equal to the distance from the ionizing source: $\mathrm{r} \leq L_{i o n} C_{v}(\mathrm{r}) /\left(\xi N_{H}\right)$, where $C_{v}(\mathrm{r})$ is the volume filling factor. For the parameters of the X-ray absorption derived from the 2000 epoch Chandra observation of NGC 7469 (Blustin et al. 2003), Blustin et al. (2005) derive $C_{v}(\mathrm{r})=0.086 \%$ and $r \leq 1.6 \mathrm{pc}$ for the X-ray absorber, which we argue is the same as Component 1 of the UV absorption. Using the parameters derived from the UV absorption, and assuming the same volume filling factor as Blustin et al. (2005), we find a somewhat firmer upper limit on the distance of Component 1 from the central engine than we could place from the variability, $\mathrm{r}<15 \mathrm{pc}$. If the UV absorption arises in higher density material embedded in the X-ray warm absorber (Krolik \& Kriss 1995, 2001), its volume filling factor may be even smaller than that estimated above, and the radial distance correspondingly smaller.

Blustin et al. (2005) found from their comprehensive analysis of the warm absorber data in the literature that most absorbers in Seyfert galaxies are located at distances from the central engine consistent with the torus, indicating that the outflow may originate there in a scenario similar to that proposed by Krolik \& Kriss $(1995,2001)$. In the case of NGC 7469, they found that the warm absorber analyzed by Blustin et al. (2003) fits into this picture. The minimum and maximum distances they estimate for the absorber indicate that it lies outside the radius of the BELR, 0.0042 pc (Wandel, Peterson, \& Malkan 1999) and within the radius of the torus, $\sim L_{44}^{0.5}$ pc (Krolik \& Kriss 2001) where $L$ is the ionizing luminosity between 1 and 1000 Ryd. Allowing for the lower ionizing luminosity relevant to the 2002 observations, a slightly larger column density weighted ionization parameter for the X-ray absorption, and a mean outflow velocity somewhat lower than measured previously by Blustin et al. (2003), $U \sim 10$ versus $U \sim 5$, and $-580 \mathrm{~km} \mathrm{~s}^{-1}$ versus $-800 \mathrm{~km} \mathrm{~s}^{-1}$, we find a similar overall result. For both the UV and X-ray absorption in Component 1, we find $r_{\min }>>r_{\text {BELR }}$, given $\mathrm{r}_{\min }=2 G M_{B H} / v_{\text {out }}^{2}$. We note, however, that because the formal uncertainties on the black hole mass from Wandel et al. (1999), $M_{B H}=0.76_{-0.76}^{+0.75} \times 10^{7} M_{\odot}$, allow the black hole mass to be as low as $1.5 \times 10^{5} M_{\odot}$, or less, making $\mathrm{r}_{\min }$ consistent with the absorber arising from an accretion disk wind. Blustin et al. (2005) propose the alternative explanation that the wind has not yet achieved the escape speed and may therefore be closer to the central engine than $r_{\min }$ as estimated above. This way, the partial covering of the continuum and/or broad line source demanded in particular by the saturated O VI profile of Component 1 may be understood.

The larger outflow velocity of Component 2 compared to Component 1 leads to a smaller lower limit on the distance of Component 2 from the central engine, $\sim 0.02$ pc. However, given the unity covering fraction of this component, it is unlikely that we can place it within the BELR, even given the uncertainty in $M_{B H}$ noted above. We use the change in $N(\mathrm{H} \mathrm{I})$ 
in Component 2 between the 1999 and 2002 epochs to estimate its maximum distance from the ionizing source from setting the recombination time to be less than the time between these observations. For a neutral fraction of $5 \times 10^{-4}, t_{\mathrm{rec}} \leq 1100$ days implies $n \gtrsim 20 \mathrm{~cm}^{-3}$. The best-fit ionization parameter for this component, $U=0.08$, implies $\mathrm{r}<600 \mathrm{pc}$.

We conclude that there is still ambiguity in the radial distances of the absorbers from the central engine of NGC 7469, but that the general picture described in previous work (Blustin et al. 2003; Kriss et al. 2003) holds: Component 1 is a high density, high ionization absorber located near or within the BELR and Component 2 is an absorber of relatively lower density and ionization located in a region that is consistent with an origin in the AGN torus.

The authors thank K. Sembach for providing the 2004 epoch STIS observations, A. Blustin for providing the 2000 epoch $X M M /$ RGS spectrum for comparison with our Chandra data, and T. Alexander for contributions to the FUSE pipeline reductions. We thank the anonymous referee for useful comments. J. E. S. acknowledges the support of a National Research Council Associateship held at NASA/Goddard Space Flight Center. J. C. L. thanks and acknowledges support from the Chandra fellowship grant PF2-30023, issued by the Chandra X-ray Observatory Center, which is operated by SAO for and on behalf of NASA under contract NAS8-39073.

\section{REFERENCES}

Adelberger, K. L., Steidel, C. C., Shapley, A. E., \& Pettini, M. 2003, ApJ, 584, 45

Arav, N., Korista, K. T., \& de Kool, M. 2002, ApJ, 566, 699

Ballantyne, D. R., \& Fabian, A. C. 2001, MNRAS, 328, L11

Bechtold, J., Dobrzycki, A., Wilden, B., Morita, M., Scott, J., Dobrzycka, D., Tran, K., \& Aldcroft, T. L. 2002, ApJS, 140, 143

Behar, E. \& Netzer, H. 2002, ApJ, 570, 165

Bianchi, S., \& Matt, G. 2002, A\&A, 387, 76

Blustin, A. J., Branduardi-Raymont, G., Behar, E., Kaastra, J. S., Kahn, S. M., Page, M. J., Sako, M., \& Steenbrugge, K. C. 2002, A\&A, 392, 453

Blustin, A. J., et al. 2003, A\&A, 403, 481 
Blustin, A. J., Page, M. J., Fuerst, S. V., Branduardi-Raymont, G., \& Ashton, C. E. 2005, A\&A, 431, 111

Brotherton, M. S., Green, R. F., Kriss, G. A., Oegerle, W., Kaiser, M. E., Zheng, W., \& Hutchings, J. B. 2002, ApJ, 565, 800

Cardelli, J. A., Clayton, G. C., \& Mathis, J. S. 1989, ApJ, 345, 245

Cavaliere, A., Lapi, A., \& Menci, N. 2002, ApJ, 581, L1

Collier, S. J., et al. 1998, ApJ, 500, 162

Collinge, M. J., et al. 2001, ApJ, 557, 2

Crenshaw, D. M. \& Kraemer, S. B. 1999, ApJ, 521, 572

Crenshaw, D. M., Kraemer, S. B., Boggess, A., Maran, S. P., Mushotzky, R. F., \& Wu, C. 1999, ApJ, 516, 750

Crenshaw, D. M., Kraemer, S. B., Hutchings, J. B., Danks, A. C., Gull, T. R., Kaiser, M. E., Nelson, C. H., \& Weistrop, D. 2000, ApJ, 545, L27

Crenshaw, D. M., et al. 2003, ApJ, 594, 116

Crenshaw, D. M., Kraemer, S. B., \& George, I. M. 2003, ARA\&A, 41, 117

De Rosa, A., Fabian, A. C., \& Piro, L. 2002, MNRAS, 334, L21

de Vaucouleurs, G., de Vaucouleurs, A., Corwin, H. G., Buta, R. J., Paturel, G., \& Fouquè, P., 1991, Third Reference Catalog of Bright Galaxies, Version 3.9 (New York: Springer Verlag)

Dickey, J. M. \& Lockman F. J. 1990, ARA\&A, 28, 215

Gabel, J. R. et al. 2005, ApJ, in press

Gabel, J. R. et al. 2003a, ApJ, 595, 120

Gabel, J. R. et al. 2003b, ApJ, 583, 178

Gehrels, N. 1986, ApJ, 303, 336

George, I. M., Turner, T. J., Netzer, H., Nandra, K., Mushotzky, R. F., \& Yaqoob, T. 1998, ApJS, 114, 73 
Granato, G. L., De Zotti, G., Silva, L., Bressan, A., \& Danese, L. 2004, ApJ, 600, 580

Grevesse, N., Noels, A., \& Sauval, A. J. 1996, ASP Conf. Ser. 99: Cosmic Abundances, 117

Hamann, F. \& Ferland, G. 1999, ARA\&A, 37, 487

Houck, J. C. \& Denicola, L. A. 2000, in ASP Conf. Ser. 216: Astronomical Data Analysis Software and Systems IX, 591

Kaastra, J. S., Mewe, R., Liedahl, D. A., Komossa, S., \& Brinkman, A. C. 2000, A\&A, 354, L83

Kaastra, J. S., Steenbrugge, K. C., Raassen, A. J. J., van der Meer, R. L. J., Brinkman, A. C., Leidahl, D. A., Behar, E., \& de Rosa, A. 2002, A\&A, 427, 2002

Kallman, T. R. 2000, XSTAR User's Guide (Greenbelt, MD: NASA/GSFC)

Kaspi, S., et al. 2001, ApJ, 554, 216

Kraemer, S. B., Crenshaw, D. M., \& Gabel, J. R. 2001, ApJ, 557, 30

Kraemer, S. B. et al. 2001, ApJ, 551, 671

Kraemer, S. B., Crenshaw, D. M., George, I. M., Netzer, H., Turner, T. J., \& Gabel, J. R. 2002, ApJ, 577, 98

Kraemer, S. B., Crenshaw, D. M., Yaqoob, T., MKernan, B., Gabel, J. R., George, I. M., Turner, T. J., \& Dunn, J. P. 2003, ApJ, 582, 125

Krolik, J. H. \& Kriss, G. A. 1995, ApJ, 447, 512

Krolik, J. H. \& Kriss, G. A. 2001, ApJ, 561, 684

Kriss, G. A. 1994, in ASP Conf. Ser. 61, Astronomical Data Analysis Software and Systems III, ed. Dr. R. Crabtree, R. J. Hanisch, \& J. Barnes (San Francisco: ASP), 437

Kriss, G. A., Davidsen, A. F., Zheng, W., Kruk, J. W., \& Espey, B. R. 1995, ApJ, 454, L7

Kriss, G. A., Blustin, A., Branduardi-Raymont, G., Green, R. F., Hutchings, J., \& Kaiser, M. E. 2003, A\&A, 403, 473

Kriss, G. A., Peterson, B. M., Crenshaw, D. M., \& Zheng, W. 2000a, ApJ, 535, 58

Kriss, G. A. et al. 2000b, ApJ, 538, L17 
Krongold, Y., Nicastro, F., Brickhouse, N. S., Elvis, M., \& Mathur, S. 2005, ApJ, 622, 842

Lee, J. C., Fabian, A. C., Brandt, W. N., Reynolds, C. S., \& Iwasawa, K. 1999, MNRAS, 310, 973

Leitherer, C., Leão, J. R. S., Heckman, T. M., Lennon, D. J., Pettini, M., \& Robert, C. 2001, ApJ, 550, 724

Makishima, K., Maejima, Y., Mitsuda, K., Bradt, H. V., Remillard, R. A., Tuohy, I. R., Hoshi, R., \& Nakagawa, M. 1986, ApJ, 308, 635

Marshall H. L., Dewey, D., \& Ishibashi, K. 2004a, SPIE, 5165, 457

Marshall H. L., Tennant A., Grant C. E., Hitchcock A. P., O’Dell S., Plucinsky P. P. 2004b, SPIE, 5165, 497

Mathur, S., Elvis, M., \& Wilkes, B. 1999, ApJ, 519, 605

Mitsuda, K., et al. 1984, PASJ, 36, 741

Nandra, K., Clavel, J., Edelson, R. A., George, I. M., Malkan, M. A., Mushotzky, R. F., Peterson, B. M., \& Turner, T. J. 1998, ApJ, 505, 594

Nandra, K., Le, T., George, I. M., Edelson, R. A., Mushotzky, R. F., Peterson, B. M., \& Turner, T. J. 2000, ApJ, 544, 734

Netzer, H., et al. 2003, ApJ, 599, 933

Ogle, P. M., Mason, K. O., Page, M. J., Salvi, N. J., Cordova, F. A., McHardy, I. M., \& Priedhorsky, W. C. 2004, ApJ, 606, 151

Reynolds, C. S. 1997, MNRAS, 286, 513

Sahnow, D. J., et al. 2000, ApJ, 538, L7

Scannapieco, E. \& Oh, S. P. 2004, ApJ, 608, 62

Schlegel, D. J., Finkbeiner, D. P., \& Davis, M. 1998, ApJ, 500, 525

Schurch, N. J., Warwick, R. S., Griffiths, R. E., \& Sembay, S. 2003, MNRAS, 345, 423

Scott, J. E., et al. 2004, ApJS, 152, 1

Steenbrugge, K. C., Kaastra, J. S., de Vries, C. P., \& Edelson, R. 2003, A\&A, 402, 477 
Wakker, B. P. et al. 2003, ApJS, 146, 1

Wakker, B. P., Kalberla, P. M. W., van Woerden, H., de Boer, K. S., \& Putman, M. E. 2001, ApJS, 136, 537

Wandel, A., Peterson, B. M., \& Malkan, M. A. 1999, ApJ, 526, 579

Wanders, I., et al. 1997, ApJS, 113, 69

Wilms, J., Allen, A., \& McCray, R. 2000, ApJ, 542, 914

Yaqoob, T., McKernan, B., Kraemer, S. B., Crenshaw, D. M., Gabel, J. R., George, I. M., \& Turner, T. J. 2003, ApJ, 582, 105

This preprint was prepared with the AAS IATEX macros v5.2. 
Table 1. Observations of NGC 7469

\begin{tabular}{lllll}
\hline \hline Instrument & \multicolumn{1}{c}{ ID } & Start Date & UT & Exp. (s) \\
\hline Chandra & 700395 & $2002-12-12$ & $13: 37: 08$ & 79840 \\
Chandra & 700586 & $2002-12-13$ & $12: 10: 13$ & 69760 \\
FUSE & C0900101 & $2002-12-13$ & $07: 07: 39$ & 3596 \\
FUSE & C0900102 & $2002-12-13$ & $06: 25: 22$ & 3352 \\
STIS & O6BN01010 & $2002-12-13$ & $06: 28: 04$ & 2233 \\
STIS & O6BN01020 & $2002-12-13$ & $07: 51: 30$ & 2695 \\
STIS & O6BN01030 & $2002-12-13$ & $09: 27: 36$ & 2695 \\
STIS & O6BN01040 & $2002-12-13$ & $11: 03: 42$ & 2695 \\
STIS & O6BN01050 & $2002-12-13$ & $12: 39: 47$ & 2695 \\
STIS & O8N501010 & $2004-06-21$ & $17: 55: 10$ & 1940 \\
STIS & O8N501020 & $2004-06-21$ & $19: 14: 52$ & 2870 \\
STIS & O8N501030 & $2004-06-21$ & $20: 50: 51$ & 2290 \\
STIS & O8N501040 & $2004-06-21$ & $22: 26: 50$ & 2870 \\
STIS & O8N501050 & $2004-06-21$ & $00: 02: 50$ & 2870 \\
STIS & O8N502010 & $2004-06-22$ & $17: 55: 3$ & 1940 \\
STIS & O8N502020 & $2004-06-22$ & $19: 14: 49$ & 2290 \\
STIS & O8N502030 & $2004-06-22$ & $20: 50: 49$ & 2870 \\
STIS & O8N502040 & $2004-06-22$ & $22: 26: 49$ & 2870 \\
\hline
\end{tabular}


Table 2. $\quad$ Spectral Features in Chandra Spectrum of NGC 7469

\begin{tabular}{|c|c|c|c|c|c|c|}
\hline$\lambda_{\mathrm{vac}}$ & $\begin{array}{c}\text { Feature } \\
(\AA)\end{array}$ & $\begin{array}{c}\text { Equiv. width } \\
(\mathrm{m} \AA)\end{array}$ & Flux $^{1}$ & $\begin{array}{c}\text { Signif. } \\
\\
\sigma\end{array}$ & $\begin{array}{c}\Delta v^{3} \\
\left(\mathrm{~km} \mathrm{~s}^{-1}\right)\end{array}$ & $\begin{array}{l}\text { FWHM } \\
\left(\mathrm{km} \mathrm{s}^{-1}\right)\end{array}$ \\
\hline \multicolumn{7}{|c|}{ Absorption } \\
\hline 6.18 & Si XIV Ly $\alpha$ & $5 \pm 2$ & & 2.3 & $-540 \pm 260$ & $620 \pm 690$ \\
\hline 6.65 & Si XIII He $\alpha$ & $11 \pm 6$ & & 5.0 & $-600 \pm 330$ & $1350 \pm 900$ \\
\hline 8.42 & $\operatorname{Mg}$ XII Ly $\alpha$ & $10 \pm 3$ & & 2.6 & $-540 \pm 100$ & $500 \pm 160$ \\
\hline 9.17 & $\operatorname{Mg}$ XI He $\alpha$ & $11 \pm 5$ & & 3.3 & $-620 \pm 130$ & $680 \pm 410$ \\
\hline 11.55 & Ne Ix $\mathrm{He} \beta$ & $14 \pm 5$ & & 4.4 & $-910 \pm 180$ & $1020 \pm 520$ \\
\hline 12.13 & $\operatorname{Ne} \mathrm{x} \operatorname{Ly} \alpha$ & $17 \pm 7$ & & $1.3^{4}$ & $-530 \pm 80$ & $430 \pm 270$ \\
\hline 12.28 & Fe XXI & $21 \pm 11$ & & $2.2^{4}$ & $-540 \pm 240$ & $890 \pm 570$ \\
\hline 12.82 & Fe $x x$ & $27 \pm 6$ & & 5.0 & $-290 \pm 130$ & $1070 \pm 340$ \\
\hline 13.45 & Ne IX $\mathrm{He} \alpha$ & $24 \pm 9$ & & 3.6 & $-670 \pm 180$ & $1050 \pm 490$ \\
\hline 13.45 & Ne IX $\mathrm{He} \alpha$ & $25 \pm 6$ & & 3.2 & $860 \pm 100$ & $760 \pm 220$ \\
\hline 15.01 & Fe XVII & $19 \pm 8$ & & 2.7 & $-690 \pm 150$ & $650 \pm 320$ \\
\hline \multicolumn{7}{|c|}{ Emission } \\
\hline & $\mathrm{Fe} \mathrm{K} \alpha$ & & $3.9 \pm 0.7$ & 17 & $6.39 \pm 0.01^{5}$ & $6310 \pm 1580$ \\
\hline 2.7 & $\ldots$ & & $1.0 \pm 0.4$ & 6.5 & 0 & $3410 \pm 1600$ \\
\hline 8.2 & $\ldots$ & & $0.8 \pm 0.4$ & 6.2 & 0 & $1600 \pm 940$ \\
\hline 11.0 & $\cdots$ & & $1.1 \pm 0.5$ & 4.5 & 0 & $590 \pm 300$ \\
\hline 12.13 & $\operatorname{Ne} \mathrm{x} \operatorname{Ly} \alpha$ & & $0.7 \pm 0.5$ & $1.3^{4}$ & $430 \pm 170$ & $480 \pm 400$ \\
\hline 13.2 & $\ldots$ & & $1.0 \pm 0.4$ & 4.5 & 0 & $790 \pm 480$ \\
\hline 13.69 & Ne IX(f) & & $1.6 \pm 0.7$ & 4.8 & $-200 \pm 290$ & $1250 \pm 690$ \\
\hline 14.45 & O VIII Ly-7? ${ }^{6}$ & & $1.0 \pm 0.4$ & 4.5 & $-180 \pm 170$ & $780 \pm 410$ \\
\hline 14.52 & O VIII Ly-6? ${ }^{6}$ & & $1.4 \pm 0.6$ & 5.9 & $810 \pm 280$ & $1270 \pm 660$ \\
\hline 14.63 & O VIII Ly-5? & & $1.5 \pm 0.8$ & 6.1 & $1470 \pm 470$ & $1790 \pm 1190$ \\
\hline 15.4 & $\ldots$ & & $1.3 \pm 0.6$ & 4.4 & 0 & $820 \pm 450$ \\
\hline 15.5 & $\ldots$ & & $1.6 \pm 0.6$ & 5.5 & 0 & $790 \pm 340$ \\
\hline 16.77 & O viI RRC & & $2.0 \pm 0.9$ & 5.8 & $-1300 \pm 230$ & $1020 \pm 530$ \\
\hline 17.1 & $\ldots$ & & $2.3 \pm 1.0$ & 6.2 & 0 & $1080 \pm 550$ \\
\hline 17.3 & $\cdots$ & & $1.5 \pm 0.8$ & 3.8 & 0 & $720 \pm 460$ \\
\hline 17.4 & $\ldots$ & & $1.4 \pm 0.7$ & 3.1 & 0 & $490 \pm 260$ \\
\hline 18.3 & $\ldots$ & & $1.9 \pm 1.0$ & 3.4 & 0 & $700 \pm 410$ \\
\hline
\end{tabular}


Table 2-Continued

\begin{tabular}{|c|c|c|c|c|c|c|}
\hline$\lambda_{\text {vac }}$ & $\begin{array}{l}\text { Feature } \\
(\AA)\end{array}$ & $\begin{array}{l}\text { Equiv. width } \\
\qquad(\mathrm{m} \AA)\end{array}$ & Flux $^{1}$ & $\begin{array}{c}\text { Signif. }{ }^{3} \\
\sigma\end{array}$ & $\begin{array}{c}\Delta v^{3} \\
\left(\mathrm{~km} \mathrm{~s}^{-1}\right)\end{array}$ & $\begin{array}{c}\text { FWHM } \\
\left(\mathrm{km} \mathrm{s}^{-1}\right)\end{array}$ \\
\hline 18.97 & O viII $\operatorname{Ly} \alpha$ & & $3.0 \pm 1.0$ & 5.1 & $50 \pm 90$ & $370 \pm 340$ \\
\hline 20.2 & $\ldots$ & & $2.4 \pm 1.5$ & 3.5 & 0 & $620 \pm 440$ \\
\hline 22.09 & O viI(f) & & $6.1 \pm 2.5$ & 3.9 & $-200 \pm 90$ & $460 \pm 220$ \\
\hline
\end{tabular}

${ }^{1}$ In units of $10^{-5}$ photons $\mathrm{cm}^{-2} \mathrm{~s}^{-1}$

${ }^{2}$ Significance of feature from smoothed equivalent width threshold. See $\S 2.2$.

${ }^{3}$ Velocity with respect to systemic redshift of NGC 7469, $z=0.01639$

${ }^{4} \mathrm{Ne} \mathrm{X}$ absorption and emission are detected in the MEG spectrum at $4.1 \sigma$ and $3.6 \sigma$, respectively, and Fe XXI absorption is detected in the MEG spectrum at 3.4 $\sigma$.

${ }^{5}$ Rest-frame energy of profile center in $\mathrm{keV}$

${ }^{6}$ ID highly uncertain, no $\operatorname{Ly} \beta, \operatorname{Ly} \gamma$, or $\operatorname{Ly} \delta$ detected. See Figure 7 and discussion in $\S 2.2$. 
Table 3. Emission Line Fits to FUSE and STIS Spectra of NGC 7469

\begin{tabular}{|c|c|c|c|c|}
\hline Line & $\begin{array}{l}\lambda_{\mathrm{vac}} \\
(\AA)\end{array}$ & Flux $^{1}$ & $\begin{array}{l}\text { Velocity }^{2} \\
\left(\mathrm{~km} \mathrm{~s}^{-1}\right)\end{array}$ & $\begin{array}{l}\text { FWHM } \\
\left(\mathrm{km} \mathrm{s}^{-1}\right)\end{array}$ \\
\hline \multicolumn{5}{|c|}{2002 FUSE } \\
\hline O vi broad & 1031.93 & $3.77 \pm 0.31$ & $-137 \pm 180$ & $5907 \pm 380$ \\
\hline O vi broad & 1037.62 & $1.88 \pm 0.16$ & $-137 \pm 180$ & $5907 \pm 380$ \\
\hline O vi narrow & 1031.93 & $2.35 \pm 0.31$ & $-162 \pm 54$ & $1061 \pm 95$ \\
\hline O vi narrow & 1037.62 & $1.17 \pm 0.16$ & $-162 \pm 54$ & $1061 \pm 95$ \\
\hline \multicolumn{5}{|c|}{2002 STIS } \\
\hline Ly $\alpha$ broad & 1215.67 & $17.59 \pm 0.31$ & $-67 \pm 89$ & $13170 \pm 130$ \\
\hline Ly $\alpha$ int. & 1215.67 & $17.13 \pm 0.49$ & $-202 \pm 63$ & $4651 \pm 131$ \\
\hline Ly $\alpha$ narrow1 & 1215.67 & $4.89 \pm 0.25$ & $-319 \pm 22$ & $1047 \pm 55$ \\
\hline Ly $\alpha$ narrow2 & 1215.67 & $3.26 \pm 0.52$ & $408 \pm 50$ & $1586 \pm 203$ \\
\hline $\mathrm{N}$ v int. & 1240.15 & $5.22 \pm 0.05$ & $-107 \pm 33$ & $4651 \pm 131$ \\
\hline N V narrow1 & 1240.15 & $0.66 \pm 0.06$ & $-107 \pm 33$ & $1047 \pm 55$ \\
\hline Si II & 1263.31 & $0.71 \pm 0.02$ & $-4 \pm 93$ & $2723 \pm 174$ \\
\hline Si II & 1531.18 & $2.09 \pm 0.11$ & $-82 \pm 53$ & $2170 \pm 88$ \\
\hline C IV broad & 1549.05 & $21.10 \pm 0.20$ & $224 \pm 51$ & $9371 \pm 78$ \\
\hline C IV int. & 1549.05 & $15.57 \pm 0.06$ & $-105 \pm 19$ & $3504 \pm 32$ \\
\hline C IV narrow & 1549.05 & $3.36 \pm 0.19$ & $-42 \pm 12$ & $1118 \pm 47$ \\
\hline Fe II & 1608.45 & $0.33 \pm 0.09$ & $795 \pm 53$ & $2386 \pm 315$ \\
\hline He II broad & 1640.50 & $6.86 \pm 0.45$ & $-326 \pm 297$ & $11041 \pm 706$ \\
\hline He II narrow & 1640.50 & $0.89 \pm 24.41$ & $-326 \pm 297$ & $1403 \pm 188$ \\
\hline \multicolumn{5}{|c|}{2004 STIS } \\
\hline Ly $\alpha$ broad & 1215.67 & $4.84 \pm 0.06$ & $-1315 \pm 231$ & $13541 \pm 144$ \\
\hline Ly $\alpha$ int. & 1215.67 & $11.66 \pm 0.12$ & $-129 \pm 23$ & $4774 \pm 54$ \\
\hline Ly $\alpha$ narrow1 & 1215.67 & $3.77 \pm 0.11$ & $-232 \pm 16$ & $1137 \pm 19$ \\
\hline Ly $\alpha$ narrow2 & 1215.67 & $1.44 \pm 0.12$ & $449 \pm 9$ & $1566 \pm 120$ \\
\hline N v int. & 1240.15 & $3.84 \pm 0.09$ & $-38 \pm 15$ & $4774 \pm 54$ \\
\hline N V narrow1 & 1240.15 & $0.42 \pm 0.03$ & $-38 \pm 15$ & $1137 \pm 19$ \\
\hline Si II & 1263.31 & $0.60 \pm 0.04$ & $-95 \pm 117$ & $3428 \pm 276$ \\
\hline Si II & 1531.18 & $0.95 \pm 0.04$ & $-82 \pm 53$ & $2844 \pm 182$ \\
\hline C IV broad & 1549.05 & $12.13 \pm 0.27$ & $381 \pm 77$ & $9069 \pm 149$ \\
\hline
\end{tabular}


Table 3-Continued

\begin{tabular}{lcccc}
\hline \hline \multicolumn{1}{c}{ Line } & $\begin{array}{c}\lambda_{\text {vac }} \\
(\AA)\end{array}$ & Flux $^{1}$ & $\begin{array}{c}\text { Velocity }^{2} \\
\left(\mathrm{~km} \mathrm{~s}^{-1}\right)\end{array}$ & $\begin{array}{c}\text { FWHM } \\
\left(\mathrm{km} \mathrm{s}^{-1}\right)\end{array}$ \\
\hline C IV int. & 1549.05 & $8.25 \pm 0.13$ & $-7 \pm 32$ & $3657 \pm 38$ \\
C IV narrow & 1549.05 & $3.97 \pm 0.12$ & $-58 \pm 18$ & $1458 \pm 17$ \\
He II broad & 1640.50 & $2.79 \pm 0.75$ & $-326 \pm 297$ & $10561 \pm 327$ \\
He II narrow & 1640.50 & $0.38 \pm 0.25$ & $-326 \pm 297$ & $1479 \pm 913$ \\
\hline
\end{tabular}

${ }^{1}$ Flux in units of $10^{-13}$ ergs $\mathrm{cm}^{-2} \mathrm{~s}^{-1}$

${ }^{2}$ Velocity relative to systemic redshift, $z=0.01639$ 
Table 4. Intrinsic Absorption in FUSE and STIS Spectra of NGC 7469

\begin{tabular}{|c|c|c|c|c|}
\hline Feature & $\begin{array}{l}\text { Comp } \\
\quad \#\end{array}$ & $\begin{array}{l}\lambda_{\text {vac }} \\
(\AA)\end{array}$ & $\begin{array}{c}N_{\text {ion }} \\
\left(10^{12} \mathrm{~cm}^{-2}\right)\end{array}$ & $\begin{array}{c}\Delta v^{1} \\
\left(\mathrm{~km} \mathrm{~s}^{-1}\right)\end{array}$ \\
\hline \multicolumn{5}{|c|}{2002} \\
\hline $\mathrm{O} \mathrm{VI}$ & $2 \mathrm{a}$ & 1031.93 & $19.2 \pm 2.0$ & $-2014 \pm 18$ \\
\hline $\mathrm{O} \mathrm{VI}$ & $2 \mathrm{~b}$ & 1031.93 & $169 \pm 17$ & $-1971 \pm 2$ \\
\hline $\mathrm{O} \mathrm{VI}$ & $2 \mathrm{c}$ & 1031.93 & $690 \pm 71$ & $-1897 \pm 2$ \\
\hline $\mathrm{O} \mathrm{vI}$ & $2 \mathrm{~d}$ & 1031.93 & $68.7 \pm 7.0$ & $-1838 \pm 18$ \\
\hline $\mathrm{O} \mathrm{VI}$ & $1 \mathrm{a}$ & 1031.93 & $160 \pm 15$ & $-730 \pm 13$ \\
\hline $\mathrm{O} \mathrm{VI}$ & $1 b$ & 1031.93 & $194 \pm 18$ & $-628 \pm 15$ \\
\hline $\mathrm{O} \mathrm{VI}$ & $1 \mathrm{c}$ & 1031.93 & $439 \pm 41$ & $-594 \pm 9$ \\
\hline $\mathrm{O} \mathrm{vI}$ & $1 d$ & 1031.93 & $753 \pm 70$ & $-546 \pm 9$ \\
\hline $\mathrm{O} \mathrm{vI}$ & $1 \mathrm{e}$ & 1031.93 & $309 \pm 29$ & $-490 \pm 12$ \\
\hline $\mathrm{O} \mathrm{VI}$ & $1 \mathrm{f}$ & 1031.93 & $97.9 \pm 9.1$ & $-483 \pm 22$ \\
\hline $\mathrm{O} \mathrm{VI}$ & $1 \mathrm{~g}$ & 1031.93 & $93.4 \pm 8.7$ & $-426 \pm 34$ \\
\hline $\mathrm{O} \mathrm{VI}$ & $1 \mathrm{~h}$ & 1031.93 & $76.8 \pm 7.1$ & $-397 \pm 23$ \\
\hline $\mathrm{O} \mathrm{VI}$ & $1 \mathrm{i}$ & 1031.93 & $107 \pm 10$ & $-320 \pm 13$ \\
\hline $\operatorname{Ly} \alpha$ & $2 \mathrm{a}$ & 1215.67 & $5.5 \pm 3.1$ & $-2005 \pm 18$ \\
\hline Ly $\alpha$ & $2 \mathrm{~b}$ & 1215.67 & $3.8 \pm 3.1$ & $-1960 \pm 39$ \\
\hline Ly $\alpha$ & $2 \mathrm{c}$ & 1215.67 & $200 \pm 58$ & $-1897 \pm 2$ \\
\hline Ly $\alpha$ & $2 \mathrm{~d}$ & 1215.67 & $19.9 \pm 4.3$ & $-1841 \pm 5$ \\
\hline Ly $\alpha$ & $1 \mathrm{a}$ & 1215.67 & $23.3 \pm 3.5$ & $-736 \pm 4$ \\
\hline Ly $\alpha$ & $1 b$ & 1215.67 & $28.1 \pm 12.4$ & $-669 \pm 15$ \\
\hline Ly $\alpha$ & $1 \mathrm{c}$ & 1215.67 & $63.6 \pm 31.4$ & $-627 \pm 14$ \\
\hline Ly $\alpha$ & $1 d$ & 1215.67 & $109 \pm 26.6$ & $-567 \pm 6$ \\
\hline $\operatorname{Ly} \alpha$ & $1 \mathrm{e}$ & 1215.67 & $44.8 \pm 9.8$ & $-504 \pm 13$ \\
\hline $\operatorname{Ly} \alpha$ & $1 \mathrm{f}$ & 1215.67 & $13.5 \pm 9.1$ & $-460 \pm 11$ \\
\hline Ly $\alpha$ & $1 \mathrm{~g}$ & 1215.67 & $14.3 \pm 10.4$ & $-422 \pm 22$ \\
\hline Ly $\alpha$ & $1 \mathrm{~h}$ & 1215.67 & $15.5 \pm 1.9$ & $-367 \pm 14$ \\
\hline Ly $\alpha$ & $1 \mathrm{i}$ & 1215.67 & $11.1 \pm 4.3$ & $-316 \pm 19$ \\
\hline $\mathrm{N} \mathrm{V}$ & $2 \mathrm{a}$ & 1238.82 & $6.7 \pm 0.3$ & $-2014 \pm 18$ \\
\hline $\mathrm{N} \mathrm{V}$ & $2 \mathrm{~b}$ & 1238.82 & $2.7 \pm 3.8$ & $-1971 \pm 2$ \\
\hline $\mathrm{N}$ V & $2 \mathrm{c}$ & 1238.82 & $242 \pm 11$ & $-1897 \pm 2$ \\
\hline
\end{tabular}


Table 4-Continued

\begin{tabular}{|c|c|c|c|c|}
\hline Feature & $\begin{array}{c}\text { Comp } \\
\#\end{array}$ & $\begin{array}{l}\lambda_{\text {vac }} \\
(\AA)\end{array}$ & $\begin{array}{c}N_{\text {ion }} \\
\left(10^{12} \mathrm{~cm}^{-2}\right)\end{array}$ & $\begin{array}{c}\Delta v^{1} \\
\left(\mathrm{~km} \mathrm{~s}^{-1}\right)\end{array}$ \\
\hline $\mathrm{N} \mathrm{V}$ & $2 \mathrm{~d}$ & 1238.82 & $24.1 \pm 1.1$ & $-1838 \pm 18$ \\
\hline $\mathrm{N} \mathrm{V}$ & $1 \mathrm{a}$ & 1238.82 & $11.4 \pm 1.7$ & $-730 \pm 13$ \\
\hline $\mathrm{N} v$ & $1 b$ & 1238.82 & $38.0 \pm 17.0$ & $-618 \pm 15$ \\
\hline $\mathrm{N} \mathrm{V}$ & $1 \mathrm{c}$ & 1238.82 & $18.4 \pm 18.6$ & $-594 \pm 9$ \\
\hline $\mathrm{N} \mathrm{V}$ & $1 \mathrm{~d}$ & 1238.82 & $53.5 \pm 8.0$ & $-546 \pm 9$ \\
\hline $\mathrm{N} \mathrm{V}$ & $1 \mathrm{e}$ & 1238.82 & $22.0 \pm 3.3$ & $-490 \pm 12$ \\
\hline $\mathrm{N} \mathrm{v}$ & 1f & 1238.82 & $6.6 \pm 1.0$ & $-483 \pm 22$ \\
\hline $\mathrm{N} \mathrm{V}$ & $1 \mathrm{~g}$ & 1238.82 & $7.0 \pm 1.0$ & $-426 \pm 34$ \\
\hline $\mathrm{N} \mathrm{V}$ & $1 \mathrm{~h}$ & 1238.82 & $5.4 \pm 0.8$ & $-397 \pm 23$ \\
\hline $\mathrm{N} \mathrm{V}$ & $1 \mathrm{i}$ & 1238.82 & $7.6 \pm 1.1$ & $-320 \pm 13$ \\
\hline $\mathrm{C}$ IV & $2 \mathrm{a}$ & 1548.20 & $5.3 \pm 0.2$ & $-2014 \pm 18$ \\
\hline C IV & $2 b$ & 1548.20 & $2.0 \pm 0.1$ & $-1971 \pm 2$ \\
\hline C IV & $2 \mathrm{c}$ & 1548.20 & $190 \pm 8$ & $-1897 \pm 2$ \\
\hline $\mathrm{C}$ IV & $2 \mathrm{~d}$ & 1548.20 & $18.9 \pm 0.8$ & $-1838 \pm 18$ \\
\hline C IV & $1 \mathrm{a}$ & 1548.20 & $1.6 \pm 0.4$ & $-730 \pm 13$ \\
\hline $\mathrm{C}$ IV & $1 b$ & 1548.20 & $5.5 \pm 1.4$ & $-618 \pm 15$ \\
\hline C IV & $1 \mathrm{c}$ & 1548.20 & $2.6 \pm 0.7$ & $-594 \pm 9$ \\
\hline C IV & $1 \mathrm{~d}$ & 1548.20 & $7.6 \pm 1.9$ & $-546 \pm 9$ \\
\hline $\mathrm{C}$ IV & $1 \mathrm{e}$ & 1548.20 & $3.1 \pm 0.8$ & $-490 \pm 12$ \\
\hline $\mathrm{C}$ IV & $1 \mathrm{f}$ & 1548.20 & $0.9 \pm 0.2$ & $-483 \pm 22$ \\
\hline $\mathrm{C}$ IV & $1 \mathrm{~g}$ & 1548.20 & $1.0 \pm 0.2$ & $-426 \pm 34$ \\
\hline C IV & $1 \mathrm{~h}$ & 1548.20 & $0.8 \pm 0.2$ & $-397 \pm 23$ \\
\hline C IV & $1 \mathrm{i}$ & 1548.20 & $1.1 \pm 0.3$ & $-320 \pm 13$ \\
\hline \multicolumn{5}{|c|}{2004} \\
\hline $\operatorname{Ly} \alpha$ & $2 \mathrm{a}$ & 1215.67 & $6.4 \pm 3.0$ & $-2005 \pm 18$ \\
\hline Ly $\alpha$ & $2 b$ & 1215.67 & $5.1 \pm 3.1$ & $-1959 \pm 38$ \\
\hline Ly $\alpha$ & $2 \mathrm{c}$ & 1215.67 & $191 \pm 36$ & $-1897 \pm 2$ \\
\hline Ly $\alpha$ & $2 \mathrm{~d}$ & 1215.67 & $36.3 \pm 5.4$ & $-1841 \pm 5$ \\
\hline Ly $\alpha$ & $1 \mathrm{a}$ & 1215.67 & $58.1 \pm 6.1$ & $-736 \pm 4$ \\
\hline $\operatorname{Ly} \alpha$ & $1 b$ & 1215.67 & $0.09 \pm 5$ & $-669 \pm 15$ \\
\hline
\end{tabular}


Table 4-Continued

\begin{tabular}{|c|c|c|c|c|}
\hline Feature & $\begin{array}{c}\text { Comp } \\
\#\end{array}$ & $\begin{array}{l}\lambda_{\text {vac }} \\
(\AA)\end{array}$ & $\begin{array}{c}N_{\text {ion }} \\
\left(10^{12} \mathrm{~cm}^{-2}\right)\end{array}$ & $\begin{array}{c}\Delta v^{1} \\
\left(\mathrm{~km} \mathrm{~s}^{-1}\right)\end{array}$ \\
\hline Ly $\alpha$ & $1 \mathrm{c}$ & 1215.67 & $304 \pm 89$ & $-627 \pm 14$ \\
\hline Ly $\alpha$ & $1 d$ & 1215.67 & & \\
\hline Ly $\alpha$ & $1 \mathrm{e}$ & 1215.67 & $83.7 \pm 10.3$ & $-504 \pm 13$ \\
\hline $\operatorname{Ly} \alpha$ & $1 \mathrm{f}$ & 1215.67 & $9.8 \pm 1.4$ & $-460 \pm 11$ \\
\hline Ly $\alpha$ & $1 \mathrm{~g}$ & 1215.67 & $31.7 \pm 4.0$ & $-422 \pm 22$ \\
\hline $\operatorname{Ly} \alpha$ & $1 \mathrm{~h}$ & 1215.67 & $14.9 \pm 3.2$ & $-367 \pm 14$ \\
\hline $\operatorname{Ly} \alpha$ & $2 \mathrm{i}$ & 1215.67 & $7.9 \pm 2.6$ & $-316 \pm 19$ \\
\hline $\mathrm{N} \mathrm{V}$ & $2 a$ & 1238.82 & $6.0 \pm$ & $-2014 \pm 18$ \\
\hline $\mathrm{N} \mathrm{v}$ & $2 b$ & 1238.82 & $8.9 \pm$ & $-1971 \pm 2$ \\
\hline $\mathrm{N} \mathrm{V}$ & $2 \mathrm{c}$ & 1238.82 & $216 \pm$ & $-1897 \pm 2$ \\
\hline $\mathrm{N} \mathrm{V}$ & $2 \mathrm{~d}$ & 1238.82 & $21.5 \pm$ & $-1838 \pm 18$ \\
\hline $\mathrm{N} \mathrm{v}$ & $1 \mathrm{a}$ & 1238.82 & $19.0 \pm$ & $-730 \pm 13$ \\
\hline $\mathrm{N} \mathrm{V}$ & $1 b$ & 1238.82 & $64.0 \pm 2$ & $-618 \pm 15$ \\
\hline $\mathrm{N} \mathrm{v}$ & & 1238.82 & 41.0 & \\
\hline $\mathrm{N} \mathrm{v}$ & $1 d$ & 1238.82 & $89.0 \pm 14.1$ & $-546 \pm 9$ \\
\hline $\mathrm{N} \mathrm{v}$ & $1 \mathrm{e}$ & 1238.82 & $36.5 \pm 5.8$ & $-490 \pm 12$ \\
\hline $\mathrm{N} \mathrm{V}$ & $1 \mathrm{f}$ & 1238.82 & $11.0 \pm 1.7$ & $-483 \pm 34$ \\
\hline $\mathrm{N} \mathrm{v}$ & $1 \mathrm{~g}$ & 1238.82 & $11.6 \pm 1.8$ & $-426 \pm 22$ \\
\hline $\mathrm{N} \mathrm{V}$ & & & & -399 \\
\hline $\mathrm{N} \mathrm{V}$ & $1 \mathrm{i}$ & 1238.82 & $12.6 \pm 2.0$ & $-320 \pm 23$ \\
\hline C IV & $2 \mathrm{a}$ & 1548.20 & $4.8 \pm 0.3$ & $-2014 \pm 18$ \\
\hline C IV & $2 \mathrm{~b}$ & 1548.20 & $1.8 \pm 0.1$ & $-1971 \pm 2$ \\
\hline C IV & $2 \mathrm{c}$ & 1548.20 & $173 \pm 12$ & $-1897 \pm 2$ \\
\hline C IV & & & & $-1838 \pm 18$ \\
\hline C IV & $1 \mathrm{a}$ & 1548.20 & $6.6 \pm 0.6$ & $-730 \pm 13$ \\
\hline $\mathrm{C}$ IV & $1 b$ & 1548.20 & $22.2 \pm 1.9$ & $-618 \pm 15$ \\
\hline C IV & $1 \mathrm{c}$ & 1548.20 & $10.8 \pm 0.9$ & $-594 \pm 9$ \\
\hline C IV & $1 \mathrm{~d}$ & 1548.20 & $30.9 \pm 2.7$ & $-546 \pm 9$ \\
\hline C IV & $1 \mathrm{e}$ & & $12.7 \pm 1.1$ & $-490 \pm 12$ \\
\hline $\mathrm{C}$ IV & $1 \mathrm{f}$ & 1548.20 & $3.8 \pm 0.3$ & $-483 \pm 22$ \\
\hline
\end{tabular}


Table 4-Continued

\begin{tabular}{lcccc}
\hline \hline Feature & $\begin{array}{c}\text { Comp } \\
\#\end{array}$ & $\begin{array}{c}\lambda_{\text {vac }} \\
(\AA)\end{array}$ & $\begin{array}{c}N_{\text {ion }} \\
\left(10^{12} \mathrm{~cm}^{-2}\right)\end{array}$ & $\begin{array}{c}\Delta v^{1} \\
\left(\mathrm{~km} \mathrm{~s}^{-1}\right)\end{array}$ \\
\hline C IV & $1 \mathrm{~g}$ & 1548.20 & $4.0 \pm 0.3$ & $-426 \pm 34$ \\
C IV & $1 \mathrm{~h}$ & 1548.20 & $3.1 \pm 0.3$ & $-397 \pm 23$ \\
C IV & $1 \mathrm{i}$ & 1548.20 & $4.3 \pm 0.4$ & $-320 \pm 13$ \\
\hline
\end{tabular}

${ }^{1}$ Velocity with respect to systemic redshift of NGC 7469, $z=0.01639$ (de Vaucouleurs et al. 1991) 


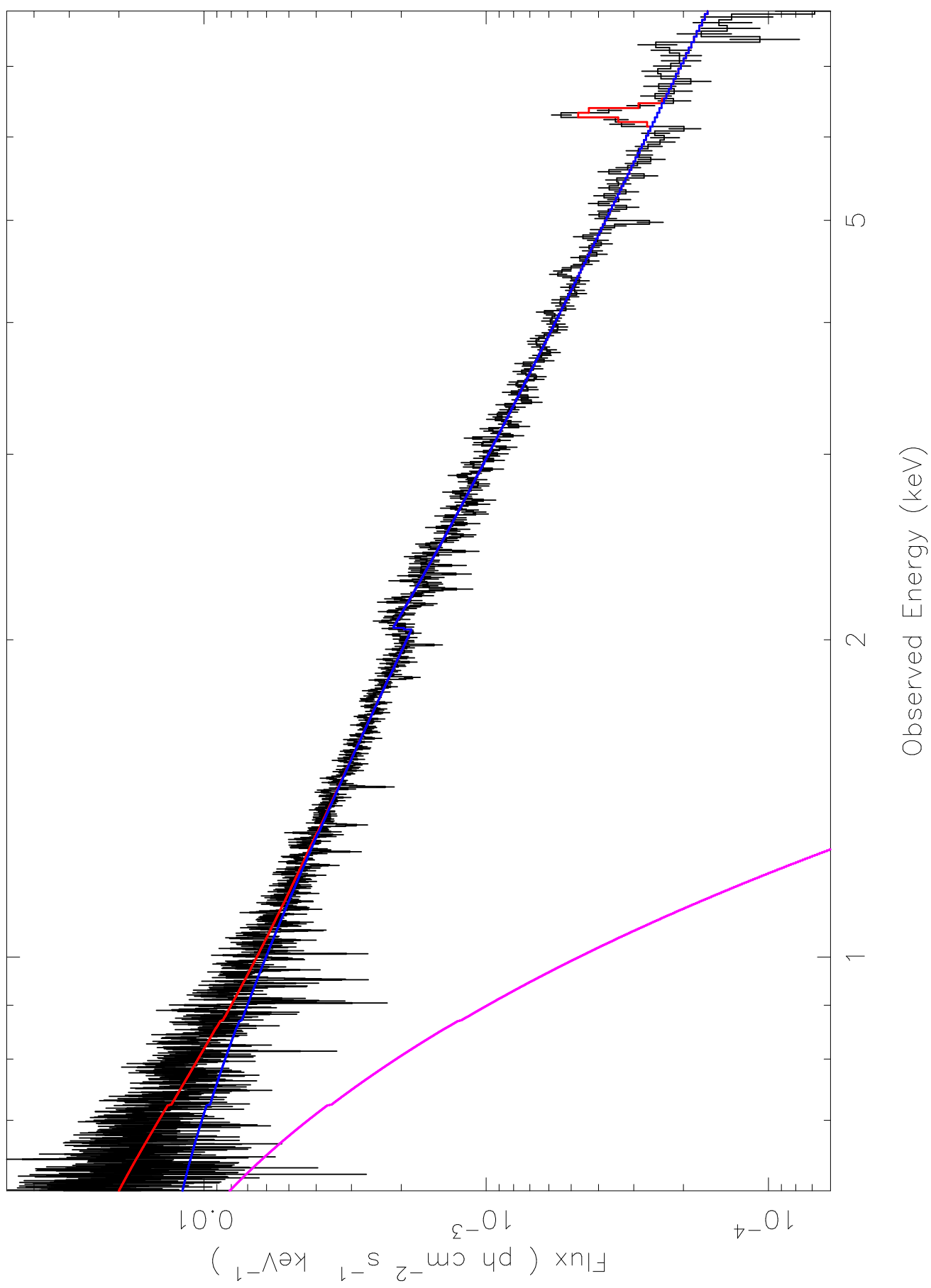

Fig. 1.- Chandra HETG spectrum of NGC 7469, binned to $0.02 \AA$, with best fit model including Fe K $\alpha$ emission (red). Power law + iridium M-edge at $5.9 \AA$ (blue) and blackbody (magenta) components are shown separately. 


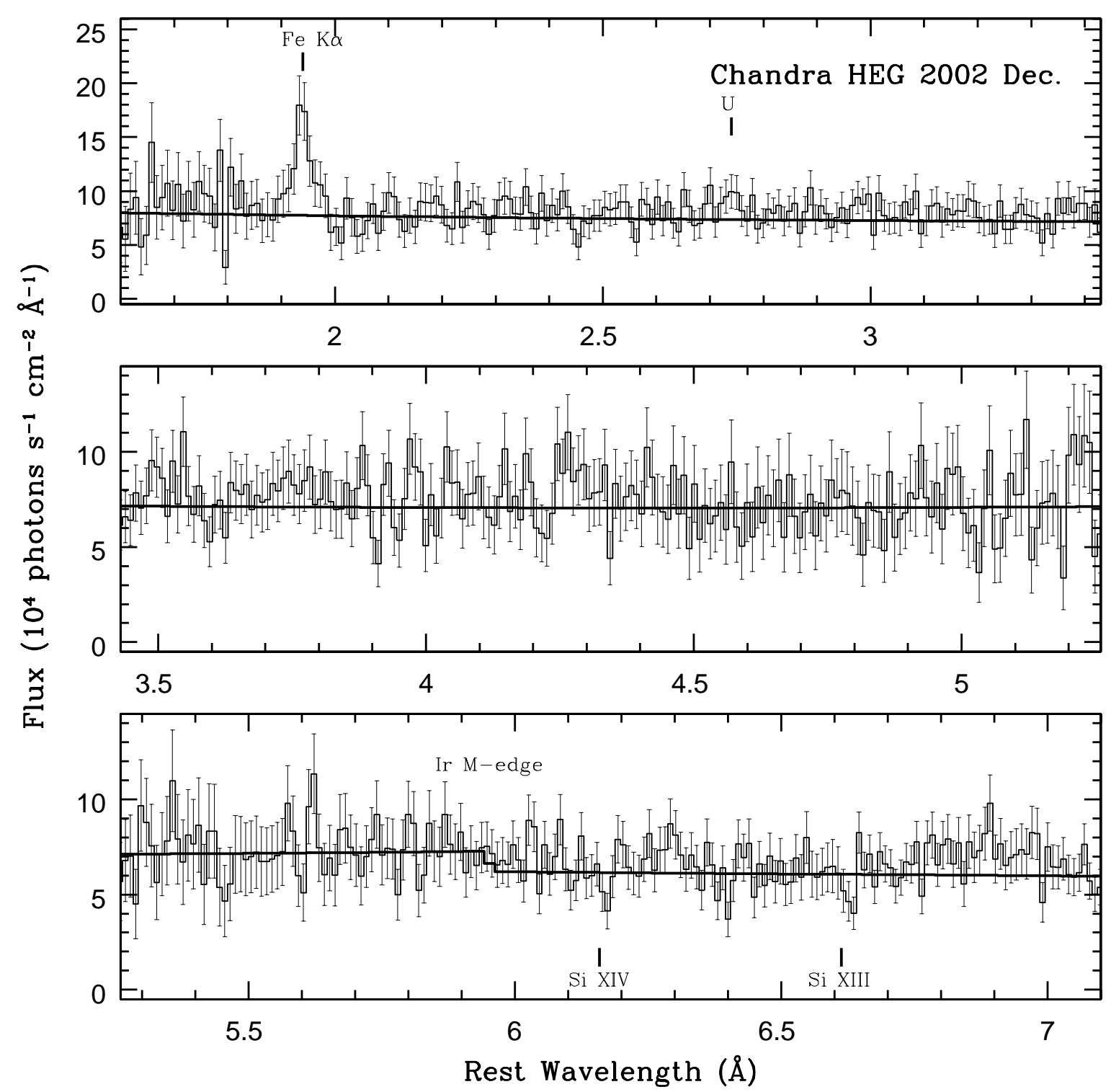

Fig. 2.- HEG spectrum of NGC 7469 with flux errors with absorption(emission) lines identified below(above) the spectrum by tick marks with labels where ID known or "U" for unidentified lines. The continuum fit in Figure 1 is shown by the solid line. 


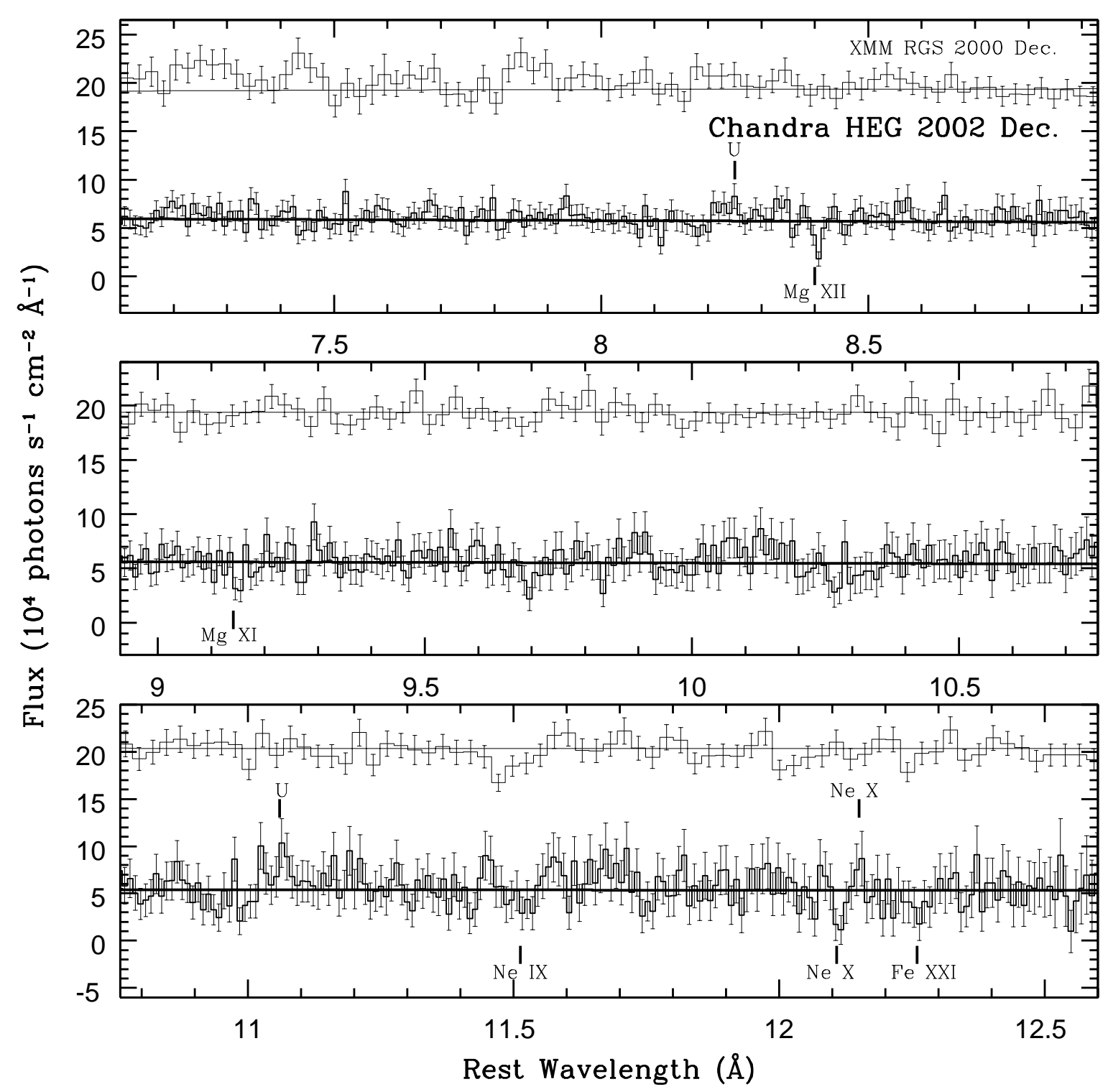

Fig. 3.- HEG spectrum of NGC 7469 with flux errors with absorption(emission) lines identified below(above) the spectrum by tick marks with labels where ID known or "U" for unidentified lines. The continuum fit in Figure 1 is shown by the solid line. Also shown is the $X M M /$ RGS spectrum obtained in 2000 by Blustin et al. (2003), offset by arbitrary factors for clarity. 


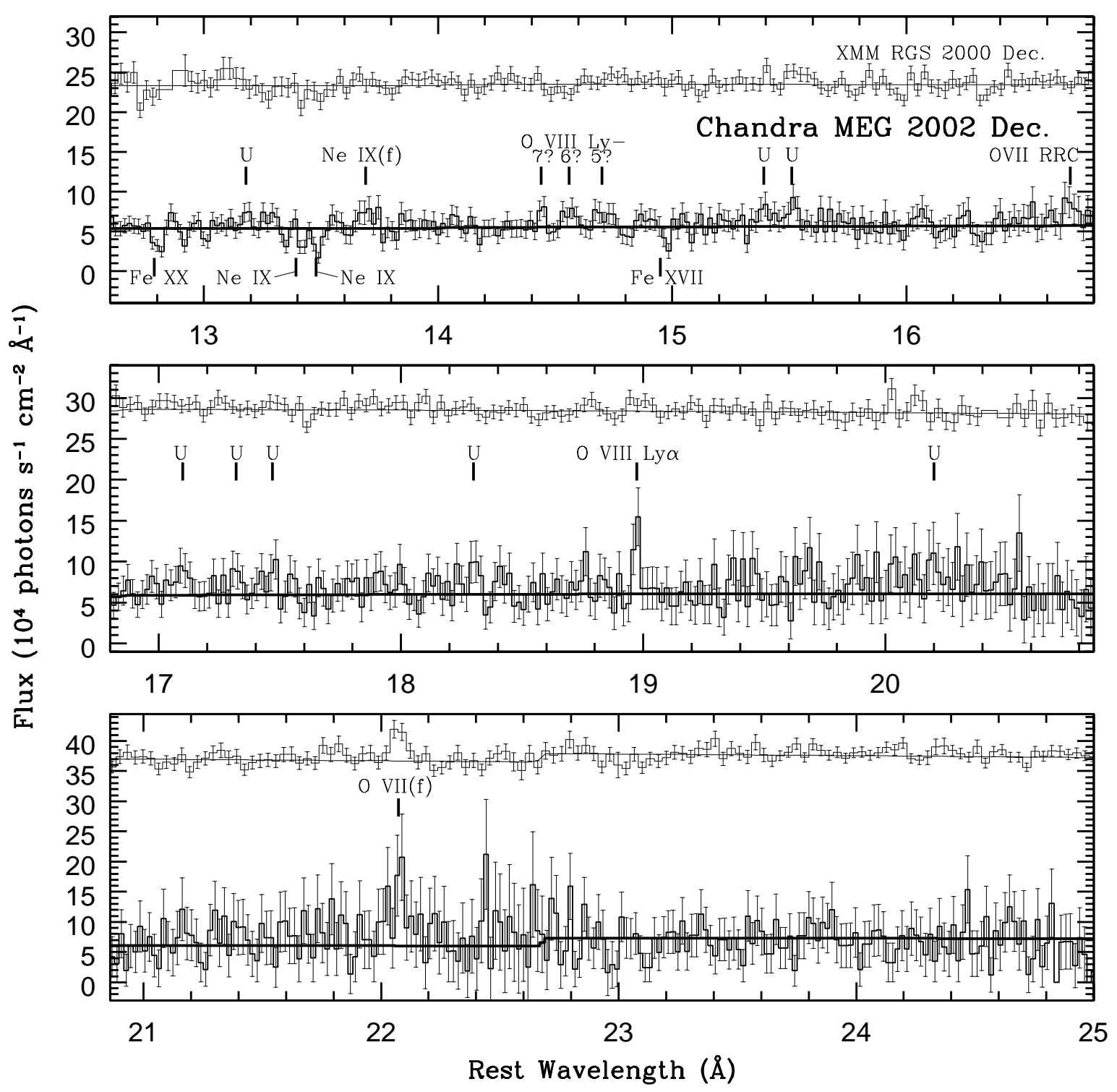

Fig. 4.- MEG spectrum of NGC 7469 with flux errors with absorption(emission) lines identified below(above) the spectrum by tick marks with labels where ID known or "U" for unidentified lines. The continuum fit in Figure 1 is shown by the solid line. Also shown is the $X M M /$ RGS spectrum obtained in 2000 by Blustin et al. (2003), offset by arbitrary factors for clarity. 


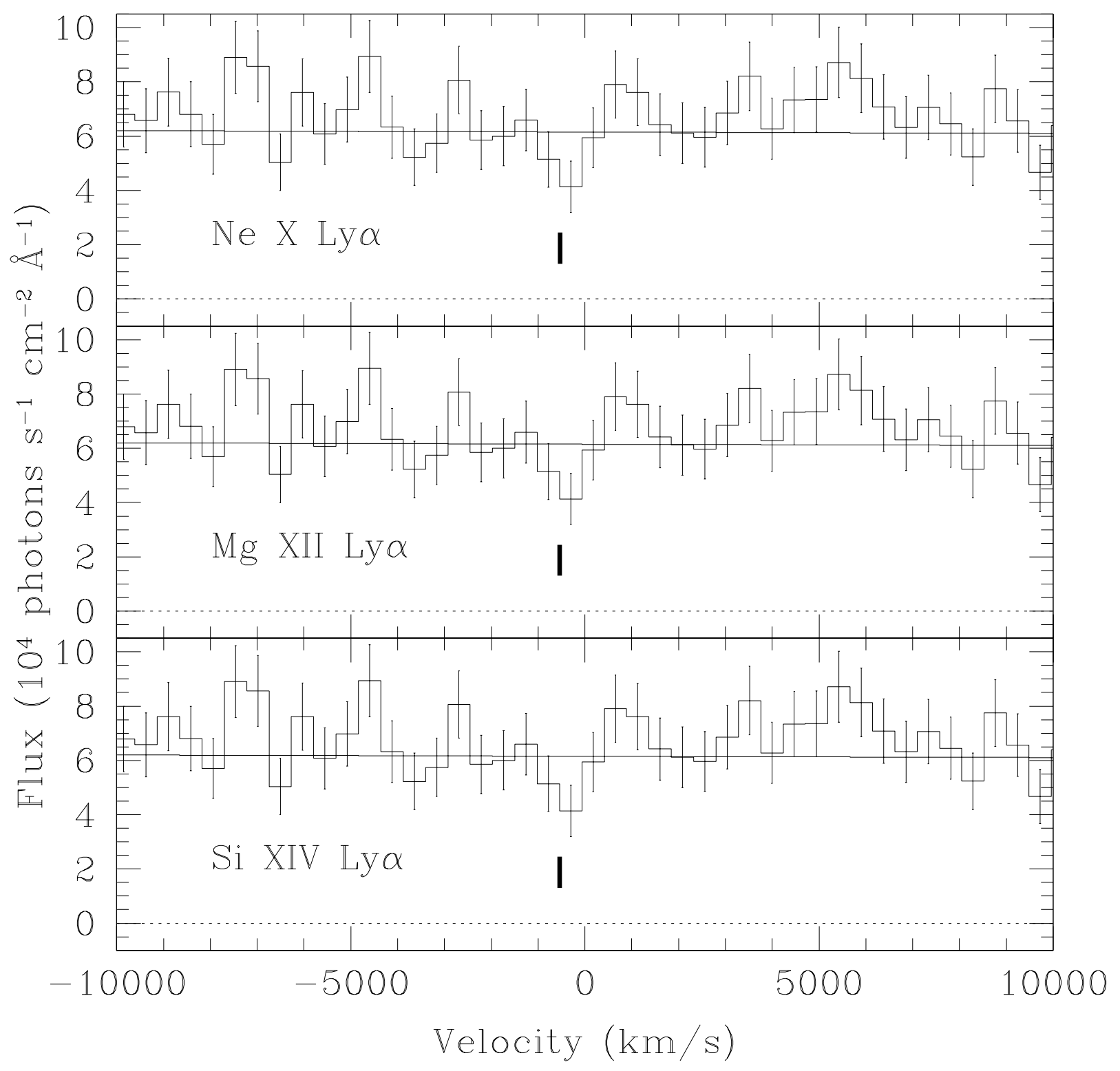

Fig. 5.- Hydrogen-like lines in Chandra/HETGS spectrum of NGC 7469, all from the HEG channel. The continuum level is the global fit shown in Figure 1. 


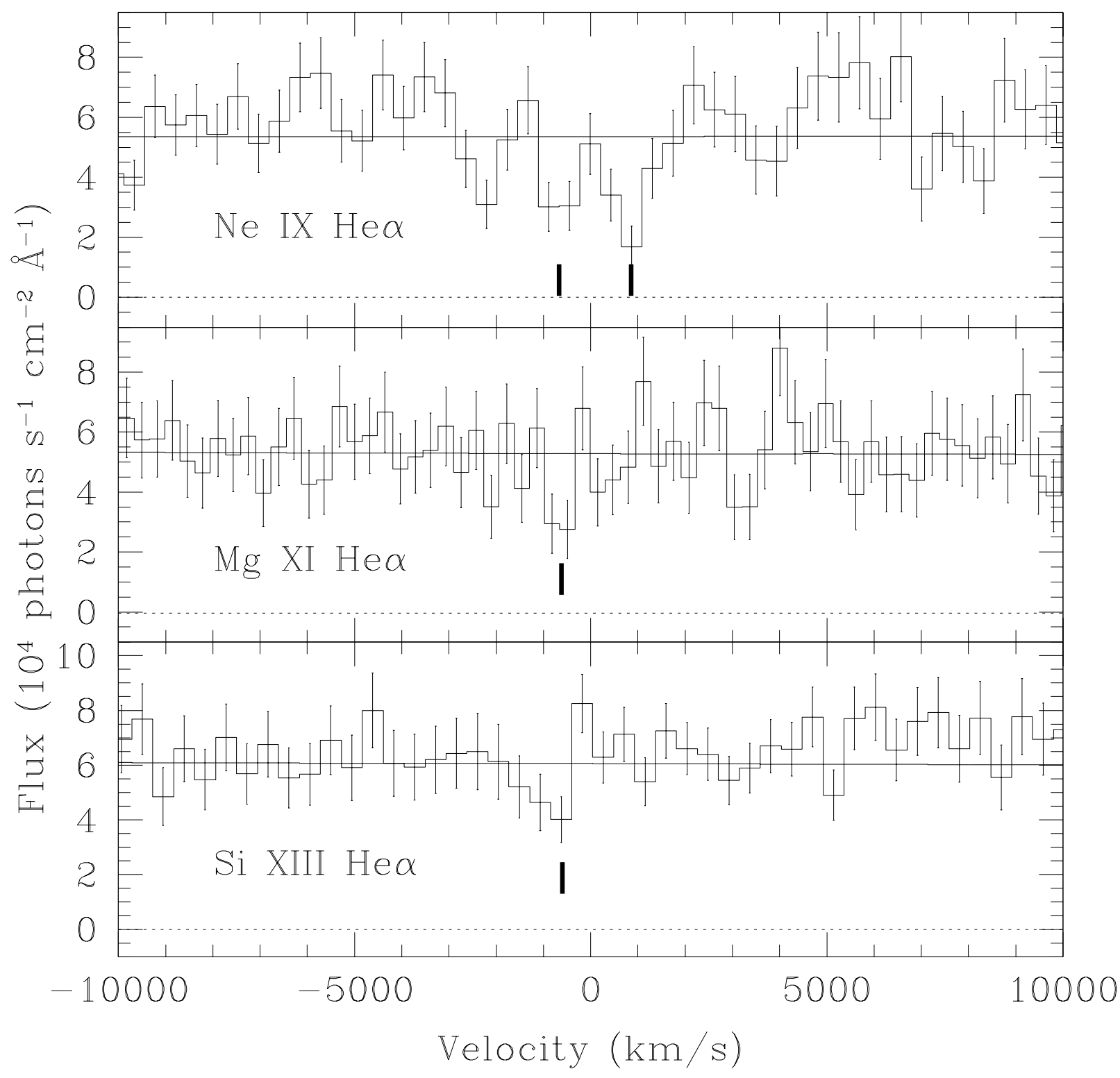

Fig. 6.- Helium-like lines in Chandra/HETGS spectrum of NGC 7469, Ne IX at $v=-590$ and $860 \mathrm{~km} \mathrm{~s}^{-1}$ from MEG, Mg XI and Si XIII from HEG channel. The continuum level is the global fit shown in Figure 1. 


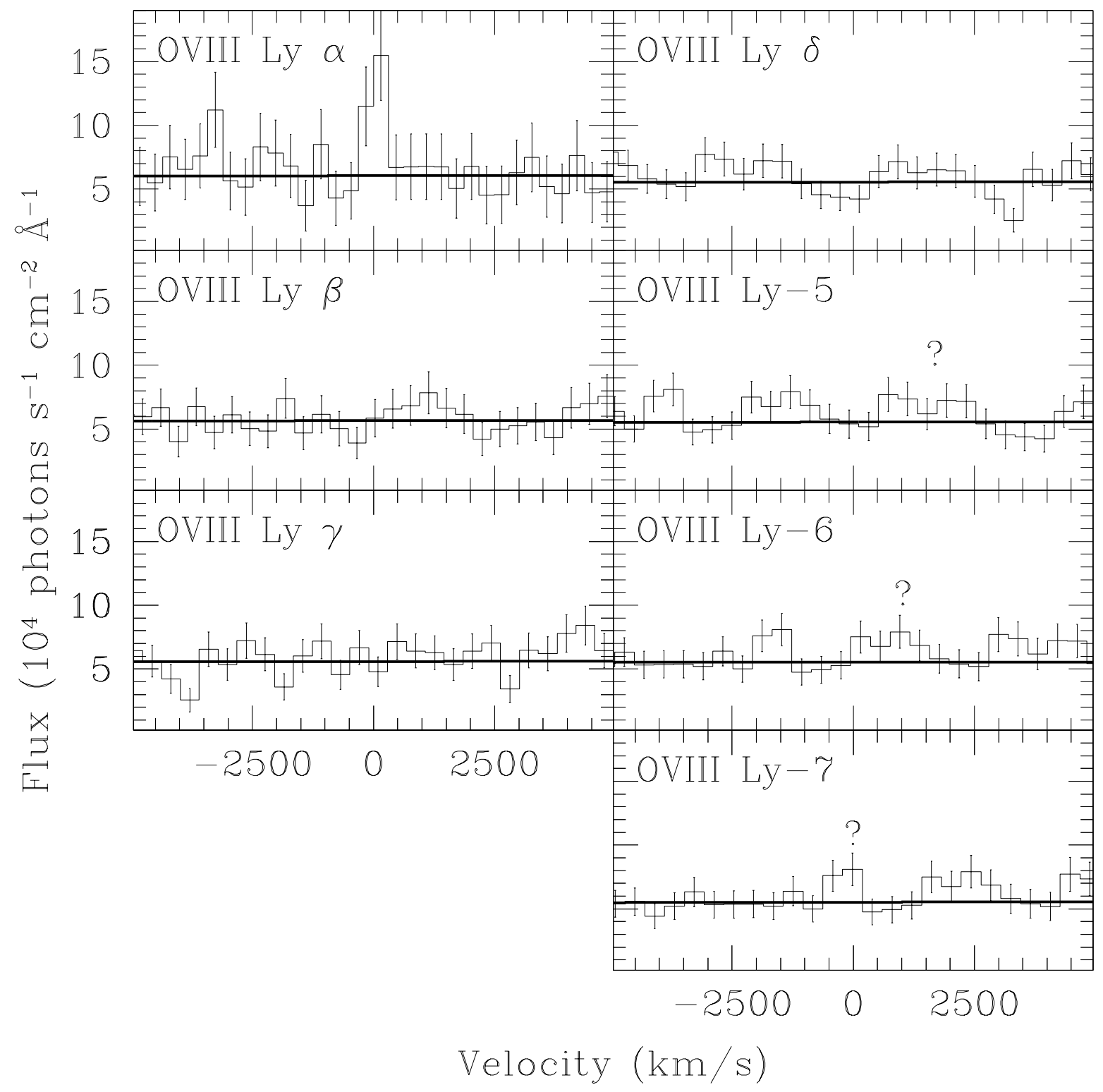

Fig. 7.- Top left panel: O VIII Ly $\alpha$ emission, detected at 3.5 $\sigma$, and possible absorption in the Chandra MEG spectrum of NGC 7469. Middle and bottom left panels and top right panel show absence of $\operatorname{Ly} \beta, \operatorname{Ly} \gamma$, and $\operatorname{Ly} \delta$ features. Middle and bottom right panels show emission features listed in Table 2 tentatively identified as O VIII Ly-5, Ly-6, and Ly-7. The continuum level in all panels is the global fit shown in Figure 1. 


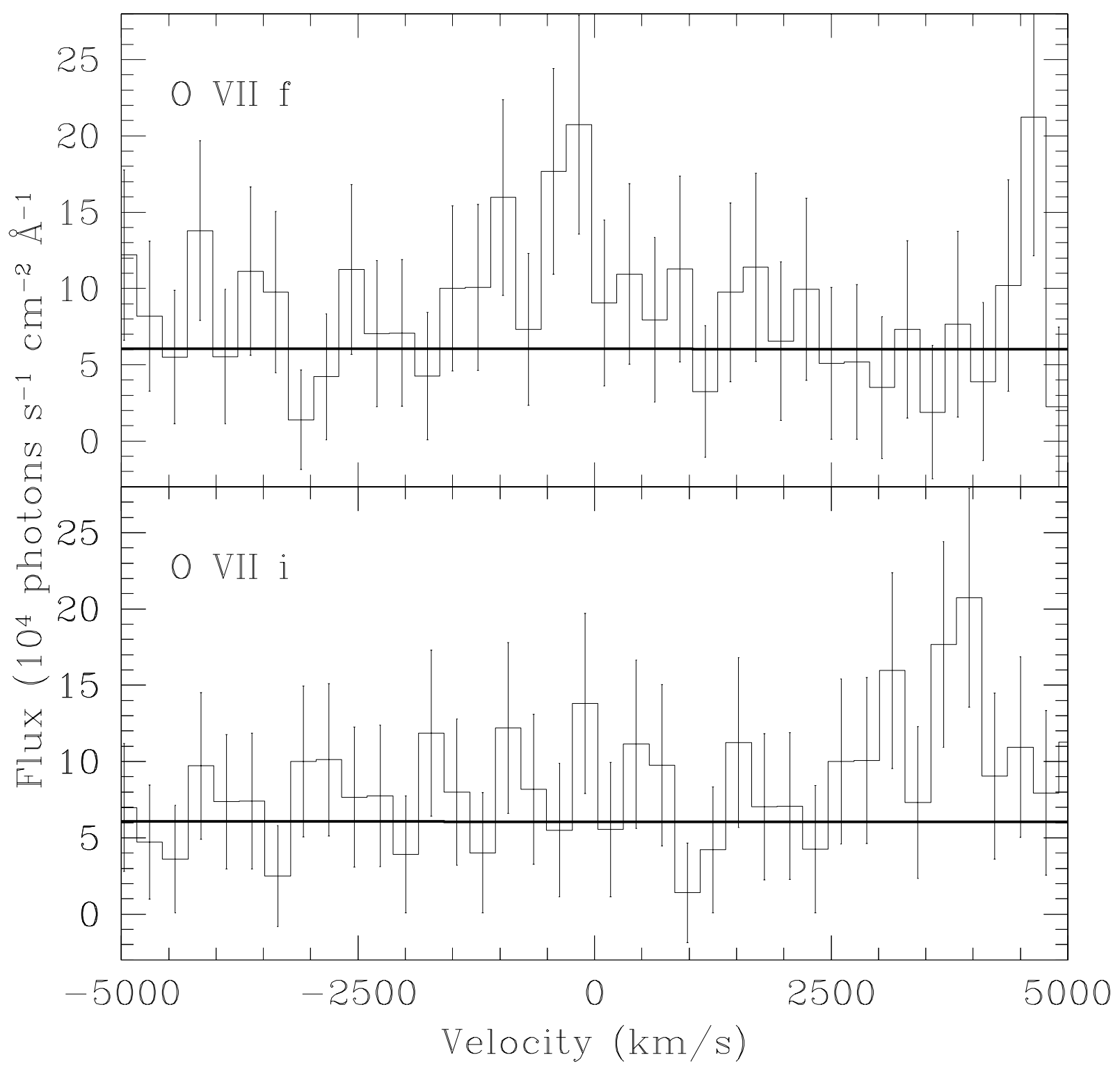

Fig. 8.- O VII forbidden emission and the expected position of corresponding intercombination emission in the Chandra MEG spectrum. The continuum level is the global fit shown in Figure 1. 


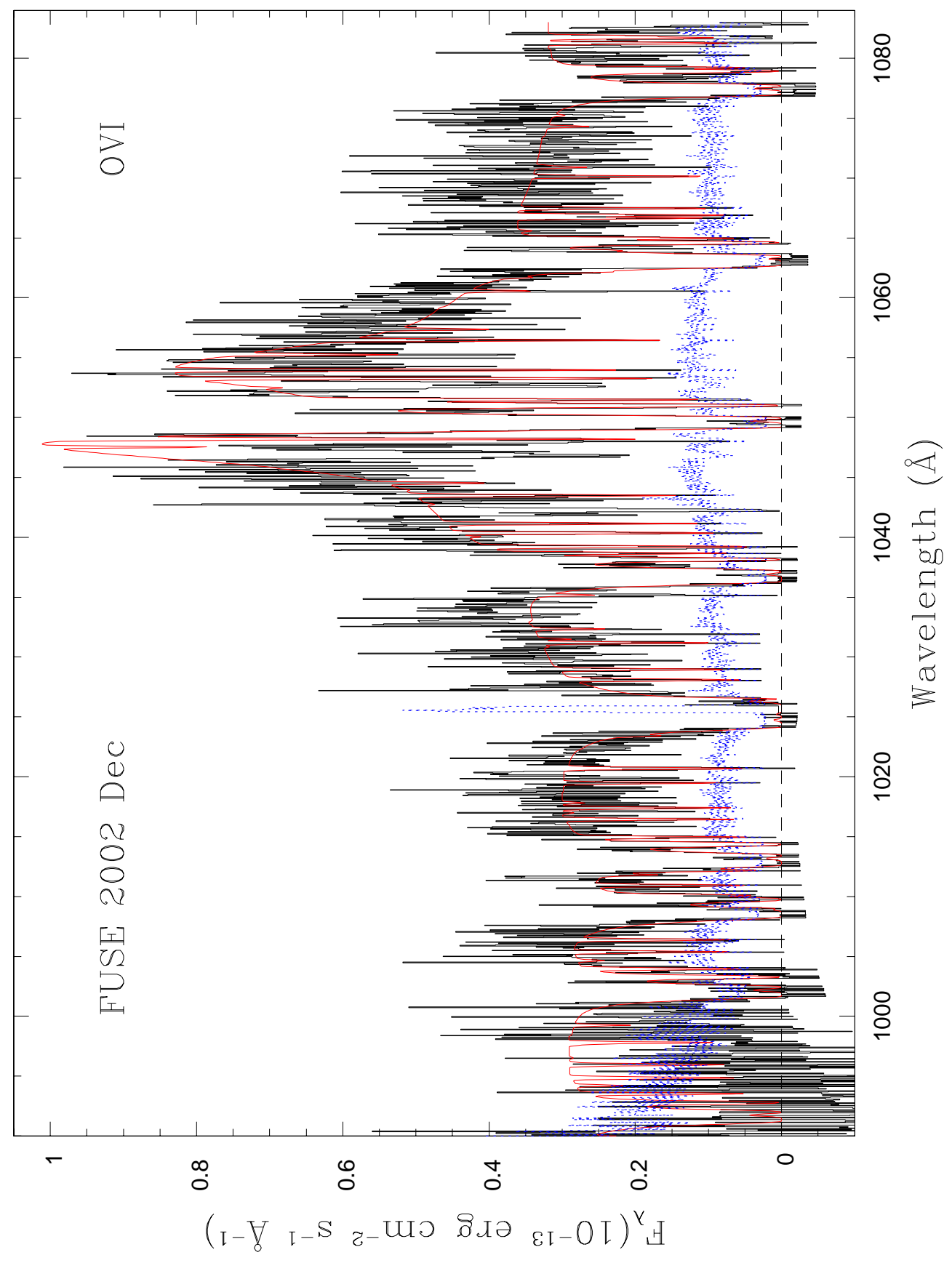

Fig. 9.- The 2002 Dec FUSE spectrum of NGC 7469 (black) with errors shown in blue, and with continuum, emission line, and ISM absorption fits (red). 

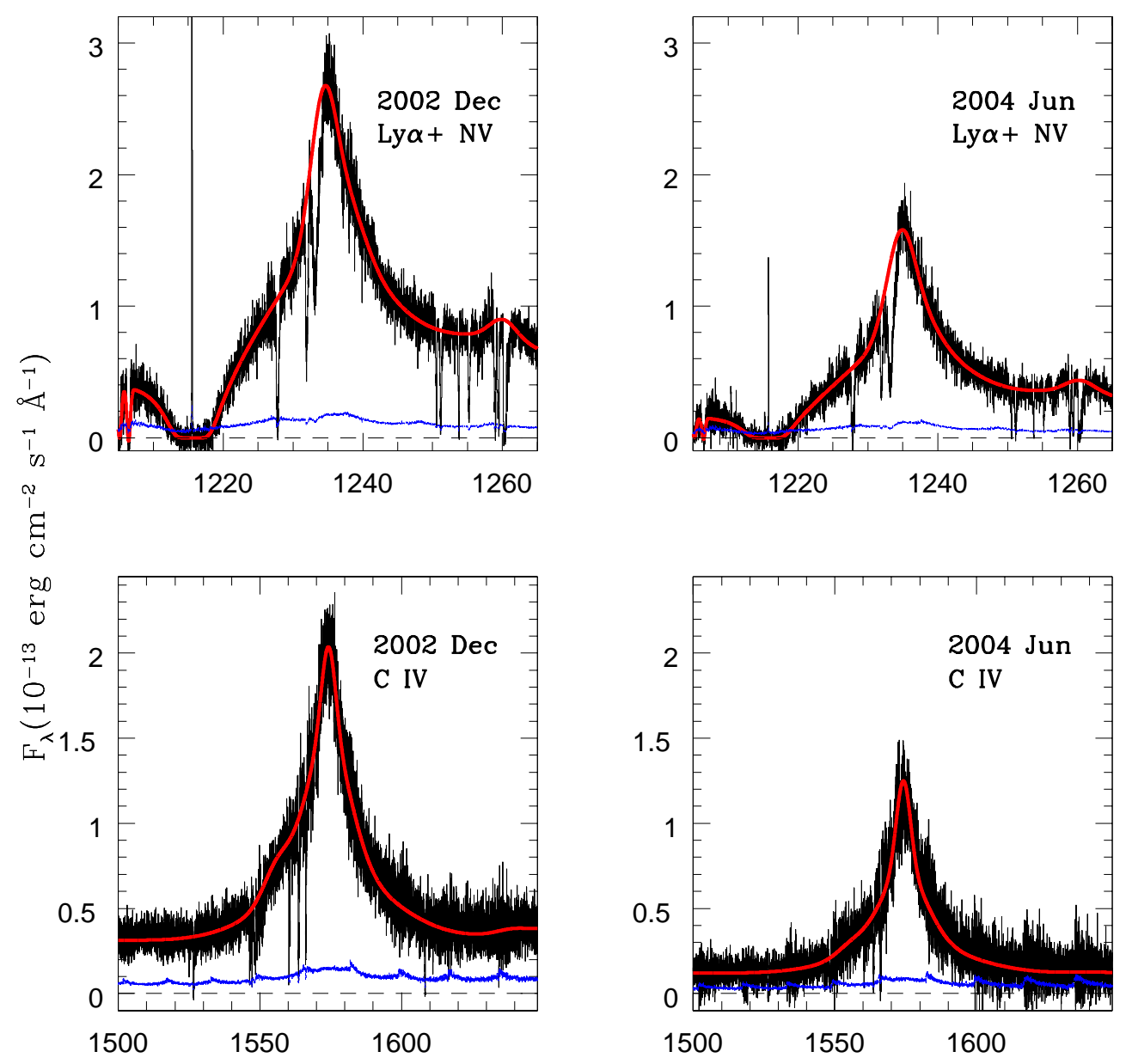

Wavelength $(\AA)$

Fig. 10.- The 2002 Dec and 2004 Jun STIS spectra of NGC 7469 (black) with errors shown in blue, and with continuum and emission line fits (red). 


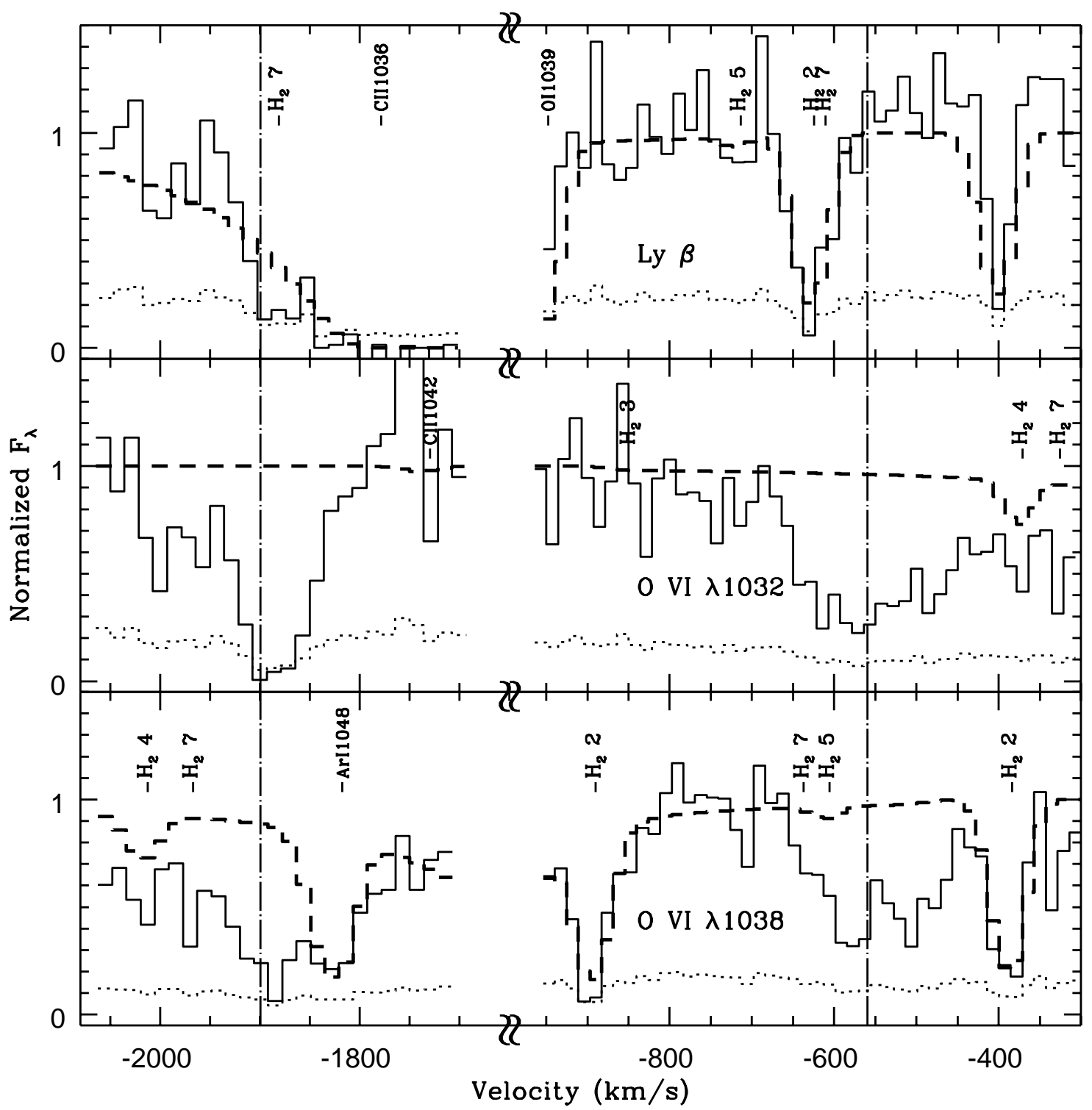

Fig. 11. - Normalized 2002 FUSE spectrum shifted to rest frame of NGC 7469, with ISM fits (dashed lines) and Ly $\beta$ and O VI intrinsic absorption. Vertical dot-dashed lines represent the column density-weighted mean velocity of all H I subcomponents in Components 1 and $2,-562 \pm 11 \mathrm{~km} \mathrm{~s}^{-1}$ and $-1901 \pm 3 \mathrm{~km} \mathrm{~s}^{-1}$, respectively. 


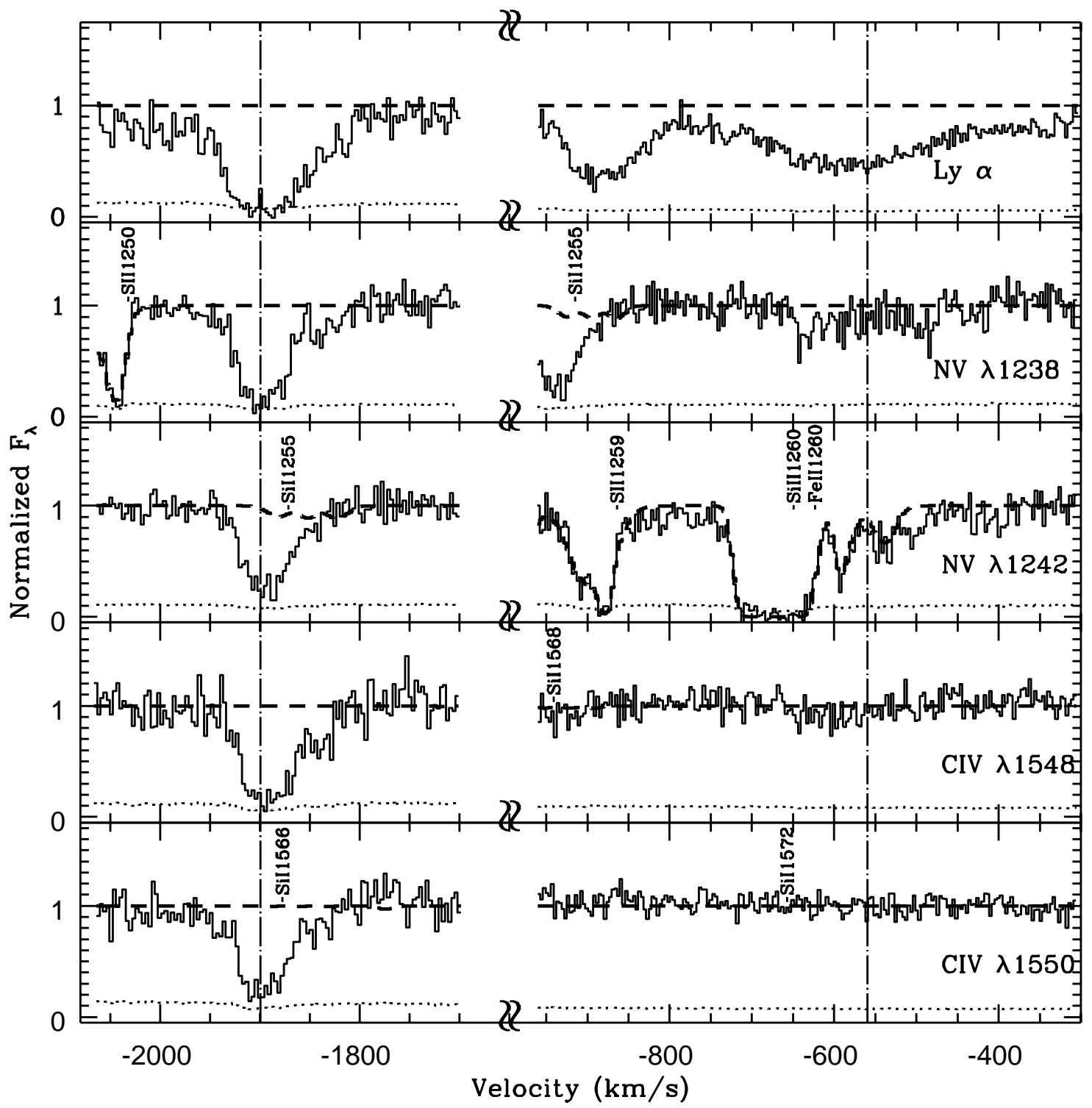

Fig. 12.- Normalized 2002 STIS spectrum shifted to rest frame of NGC 7469, with ISM fits (dashed lines) and Ly $\alpha, \mathrm{N}$ V and C IV intrinsic absorption. Vertical dot-dashed lines represent the column density-weighted mean velocity of all H I subcomponents in Components 1 and $2,-562 \pm 11 \mathrm{~km} \mathrm{~s}^{-1}$ and $-1901 \pm 3 \mathrm{~km} \mathrm{~s}^{-1}$, respectively. 


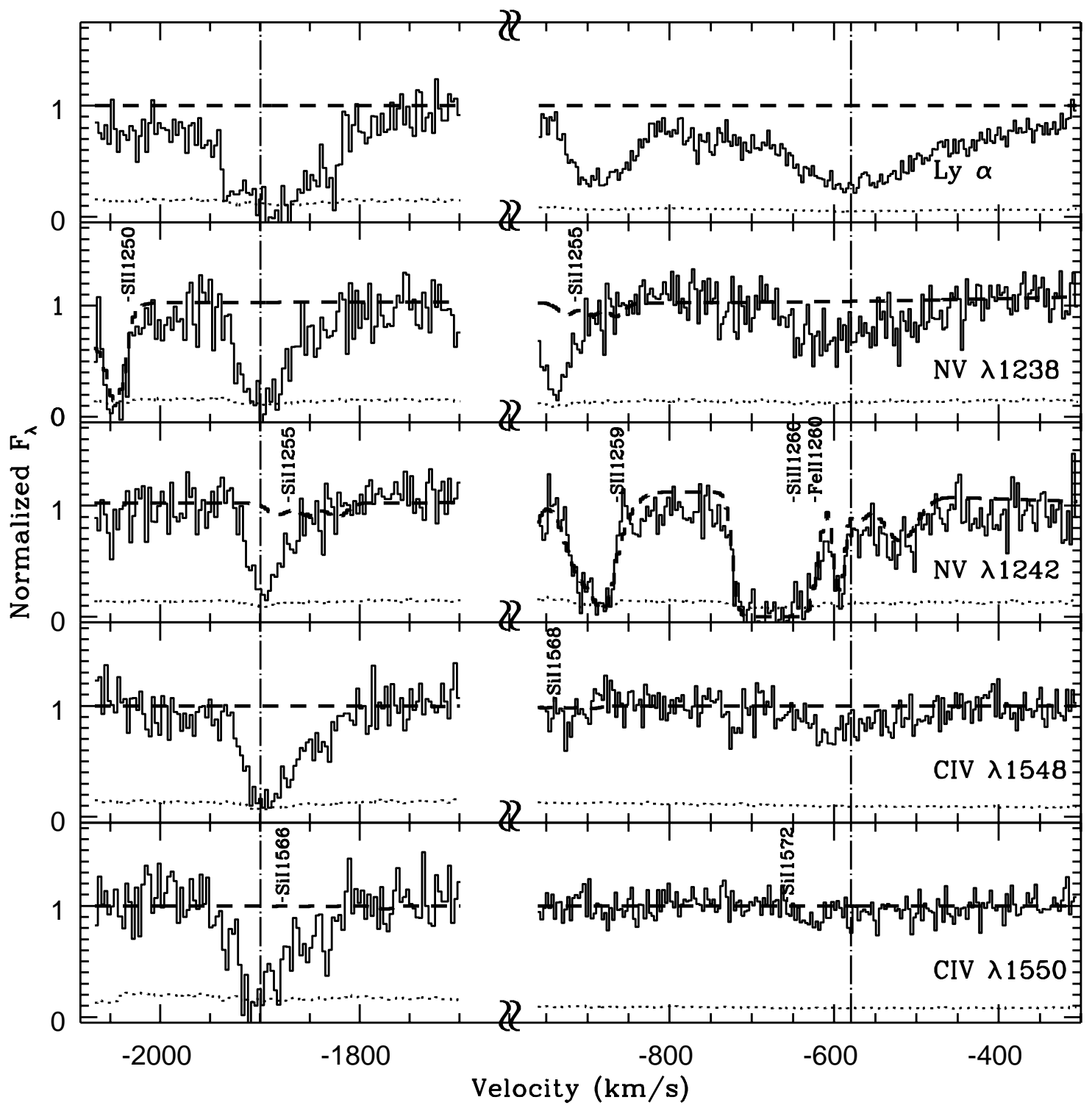

Fig. 13. - Normalized 2004 STIS spectrum shifted to rest frame of NGC 7469, with ISM fits (dashed lines) and Ly $\alpha, \mathrm{N}$ V and C IV intrinsic absorption. Vertical dot-dashed lines represent the column density-weighted mean velocity of all H I subcomponents in Components 1 and $2,-583 \pm 9 \mathrm{~km} \mathrm{~s}^{-1}$ and $-1902 \pm 3 \mathrm{~km} \mathrm{~s}^{-1}$, respectively. 


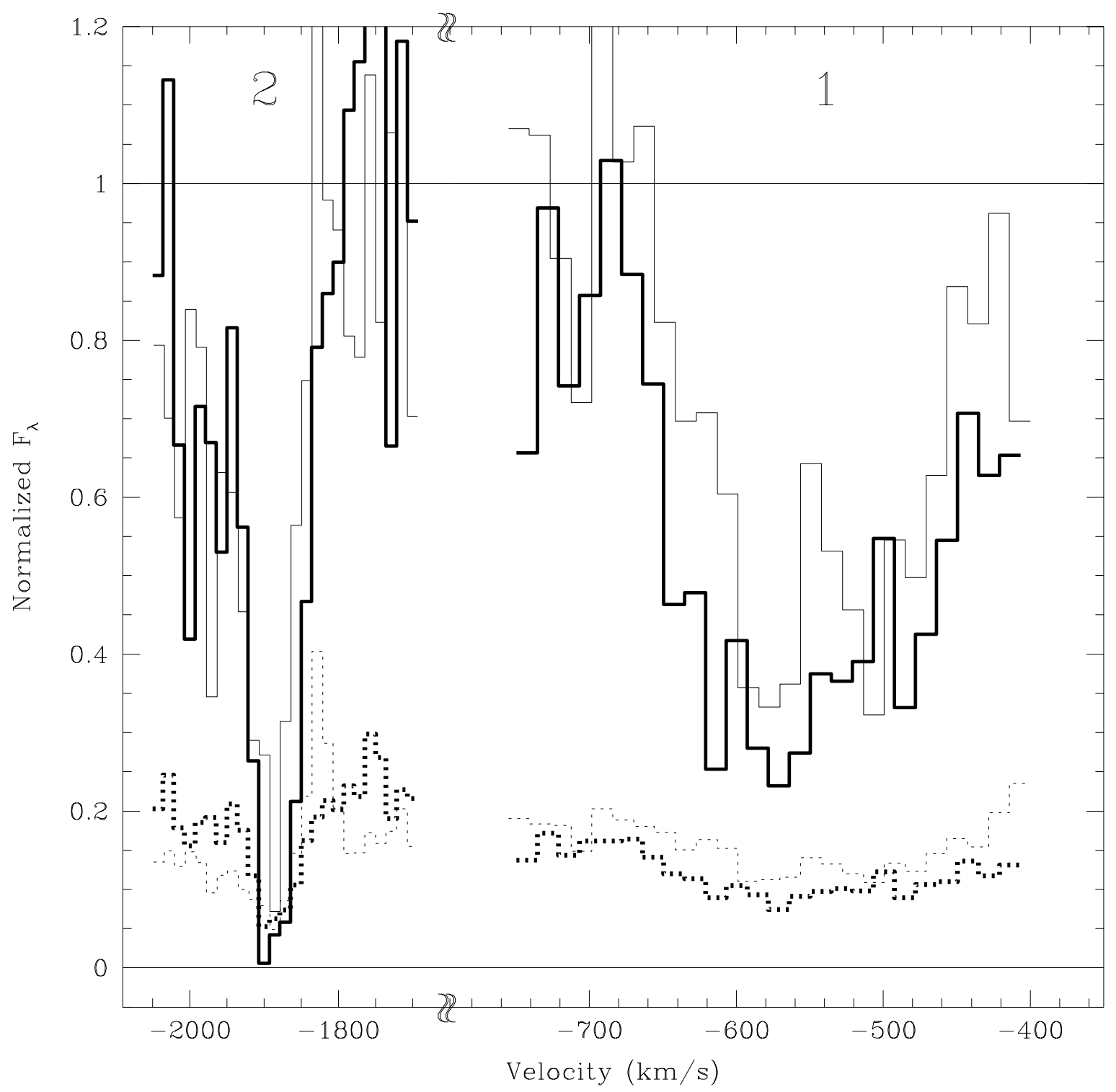

Fig. 14.- O VI doublet in Components 1 and 2. The flux in the $1032 \AA$ line is shown by the solid bold lines, errors are shown by the dotted bold lines. The $1036 \AA$ lines are shown by the regular solid and dotted lines. 


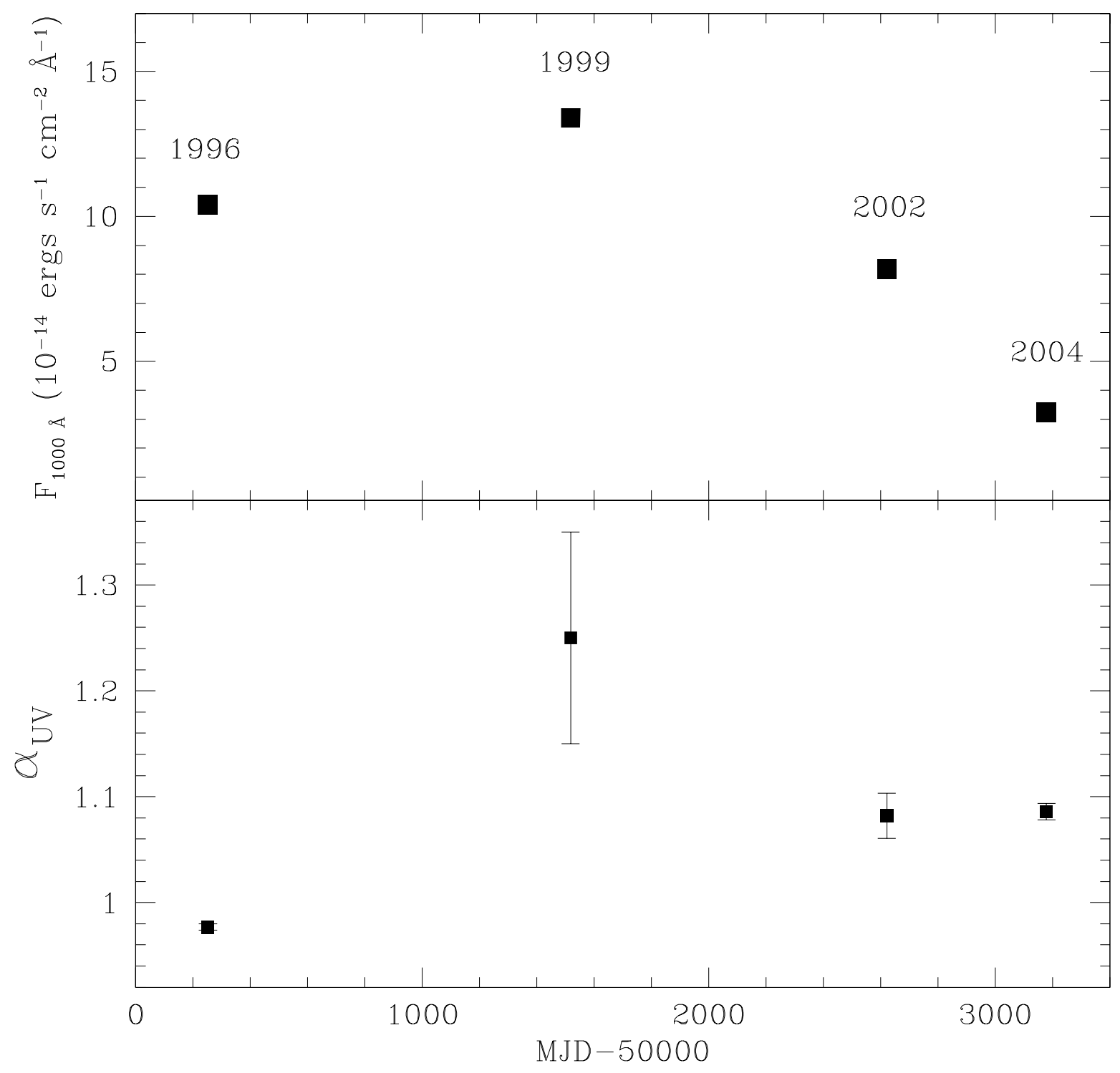

Fig. 15.- Variation in the continuum flux and the UV spectral index of NGC 7469. 

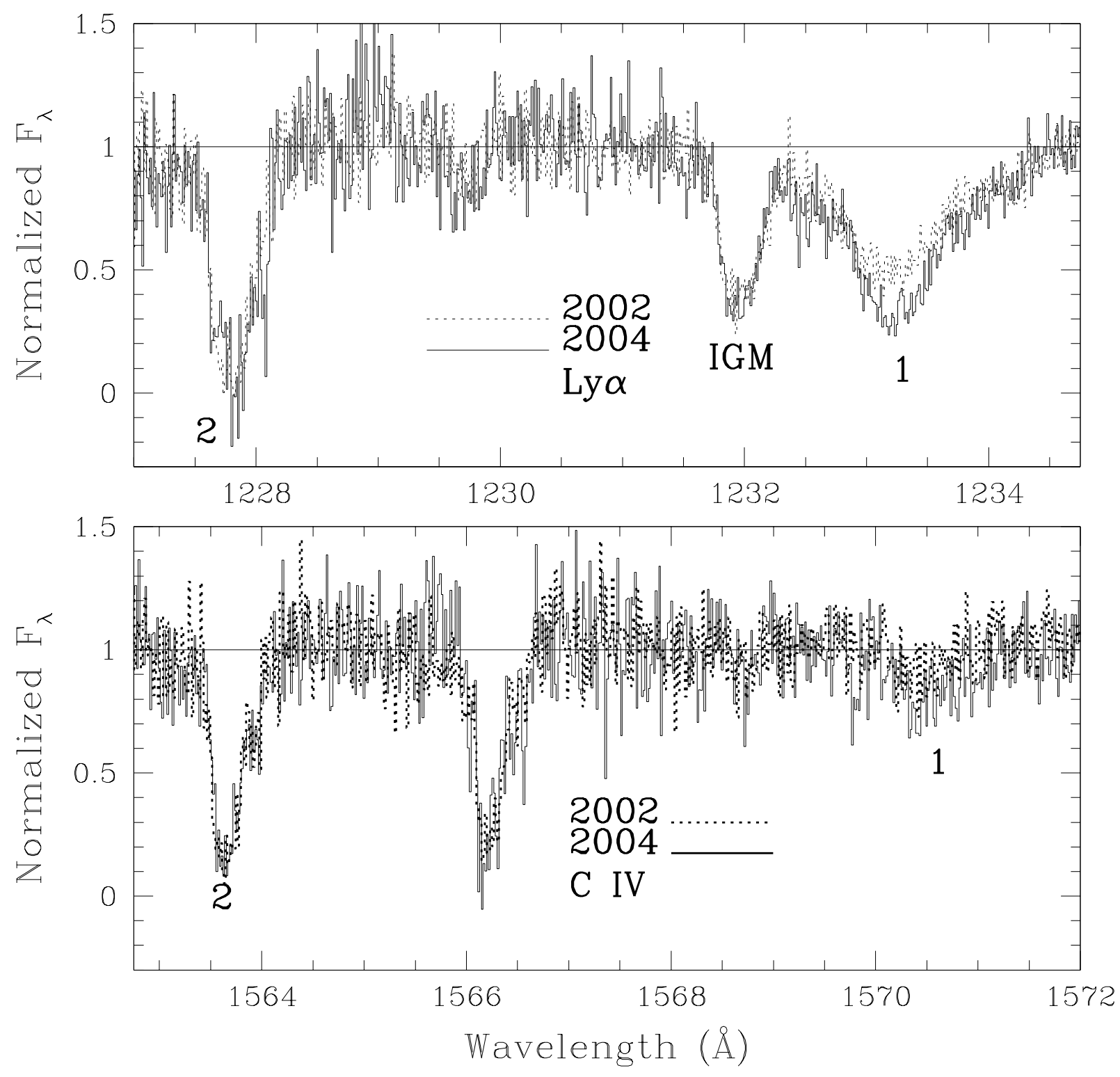

Fig. 16. - Comparison of absorption in Ly $\alpha$ and C IV between 2002 and 2004. 


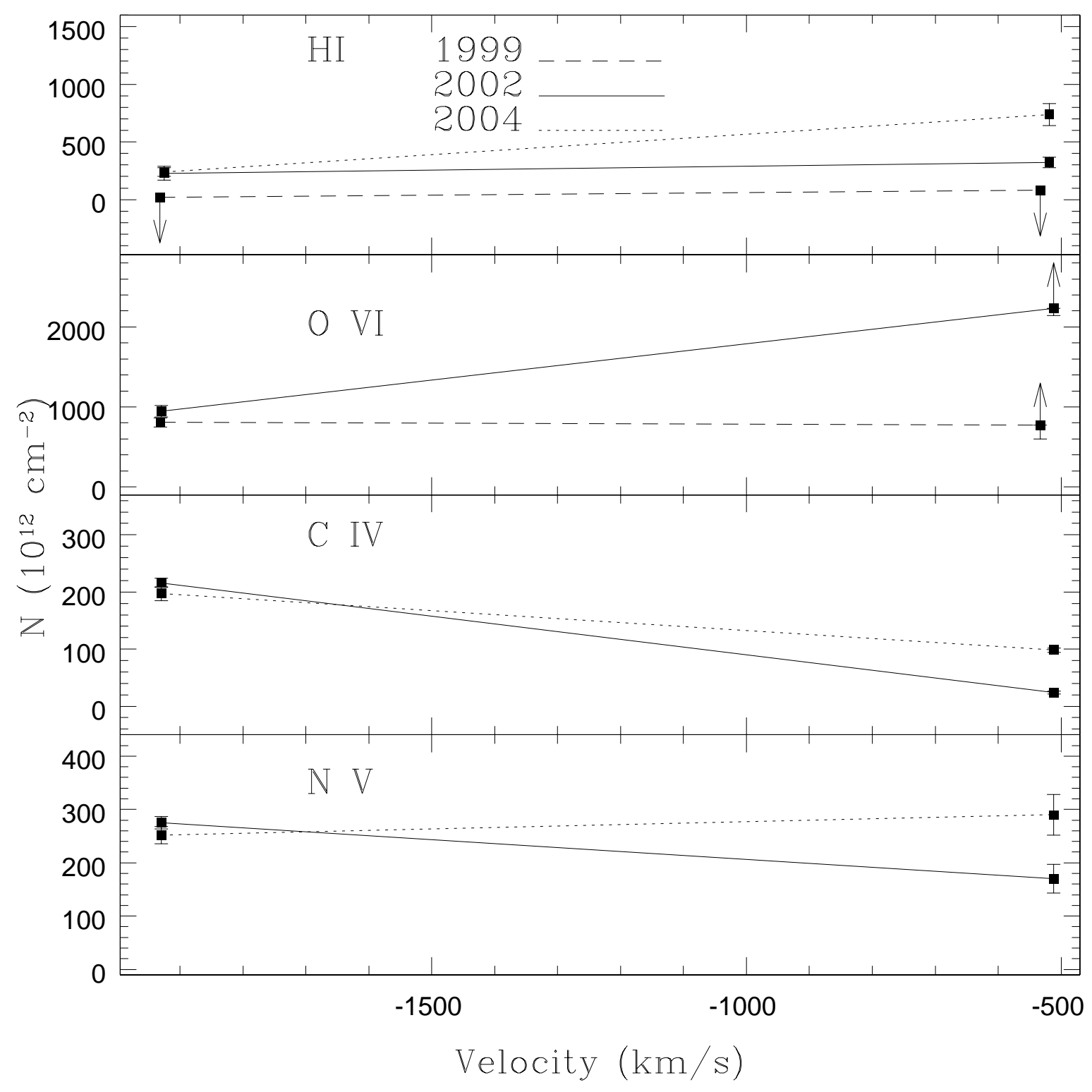

Fig. 17.- Summary of H I, O VI, C IV, and N V absorption intrinsic to NGC 7469 in 1999, 2002, and 2004 epochs. 


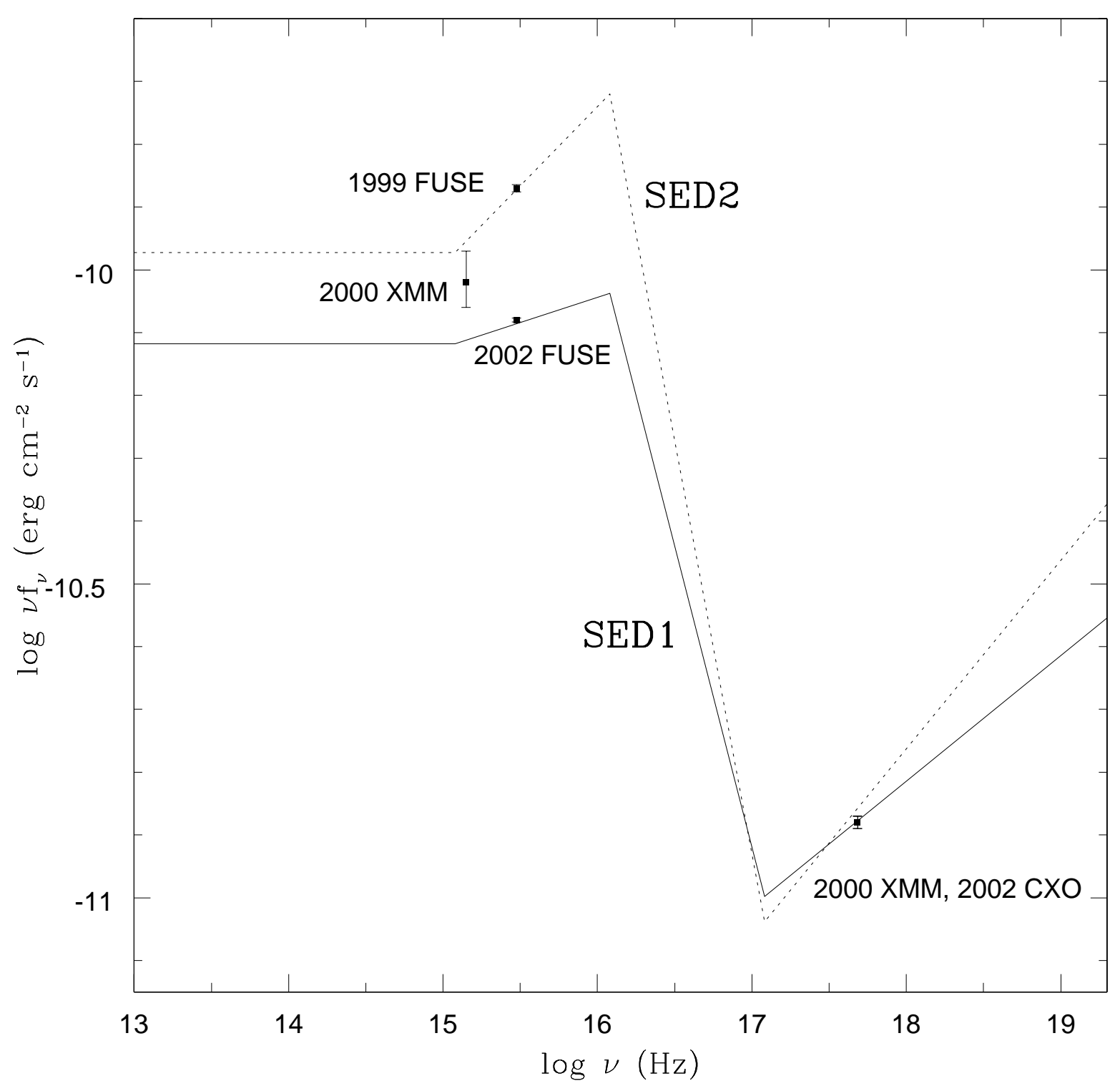

Fig. 18. - Spectral energy distributions used in photoionization models of the intrinsic absorbers in NGC 7469. SED1 is normalized such that it matches the $1000 \AA$ flux in the 2002 epoch FUSE spectrum and the $2 \mathrm{keV}$ flux in the Chandra spectrum. SED2 is normalized to the 2000 epoch FUSE and XMM-Newton data plotted, as described by Kriss et al. (2003). 


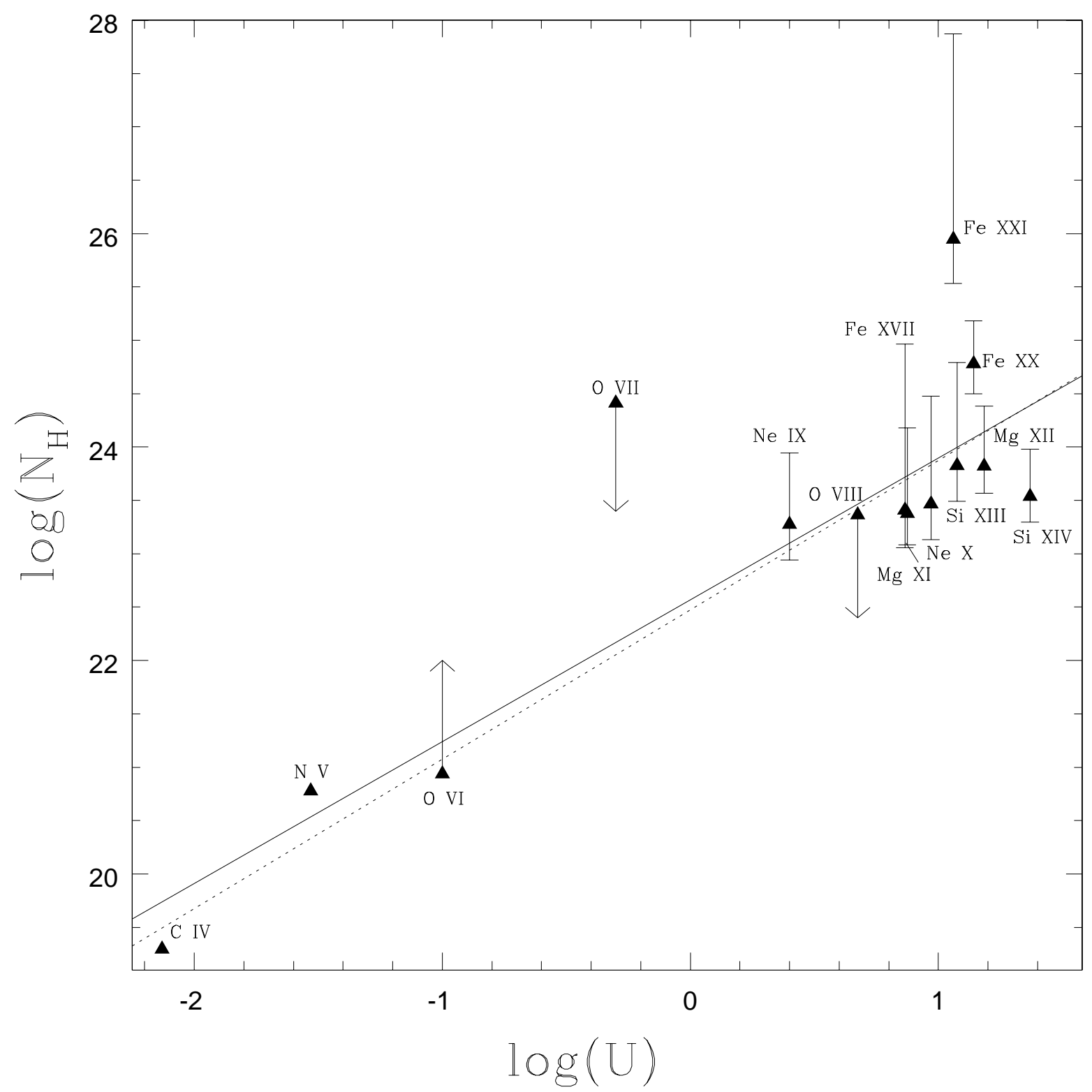

Fig. 19.- Equivalent hydrogen column density versus ionization parameter at peak abundance of each ion with power law fit. The dotted line shows a fit with the C IV, N V, and O VI points omitted. 